\title{
Enantioselective Organocatalytic Amine Conjugate Addition
}

\author{
Young K. Chen, Masanori Yoshida and David W.C. MacMillan* \\ Division of Chemistry and Chemical Engineering, California Institute of Technology, \\ Pasadena, California 91125
}

\section{Supporting Information}

General Information. Benzyl hydroxycarbamate, tert-butyl hydroxycarbamate and fluorenylmethyl hydroxycarbamate were purchased from Aldrich Chemical. All solvents were purified according to the method of Grubbs. ${ }^{1}$ All $\alpha, \beta$-unsaturated aldehydes were purified by either distillation or silica gel chromatography prior to use. Organic solutions were concentrated under reduced pressure on a Büchi rotary evaporator. Chromatographic purification of products was accomplished using flash chromatography on Silicycle 230-400 mesh silica gel according to the method of Still. Thin-layer chromatography (TLC) was carried out on Silicycle $0.25 \mathrm{~mm}$ silica gel plates. Visualization of the developed chromatogram was performed by fluorescence quenching, iodine, $p$-anisaldehyde or ceric ammonium molybdate staining.

${ }^{1} \mathrm{H}$ and ${ }^{13} \mathrm{C}$ NMR spectra were recorded on a Varian Mercury 300 Spectrometer (300 $\mathrm{MHz}$ and $75 \mathrm{MHz}$ respectively), and are internally referenced to residual protic solvent signals $\left(\mathrm{CHCl}_{3}=7.26 \mathrm{ppm}\right.$, benzene $\left.=7.16 \mathrm{ppm}\right)$. Data for ${ }^{1} \mathrm{H}$ are reported as follows: chemical shift $(\delta \mathrm{ppm})$, multiplicity $(\mathrm{s}=$ singlet, $\mathrm{d}=$ doublet, $\mathrm{t}=$ triplet, $\mathrm{q}=$ quartet, $\mathrm{m}=$ multiplet $)$, integration, coupling constant $(\mathrm{Hz})$ and assignment. Data for ${ }^{13} \mathrm{C}$ NMR are reported in terms of chemical shift. IR spectra were recorded on a Perkin Elmer Paragon 1000 spectrometer and are reported in terms of frequency of absorption $\left(\mathrm{cm}^{-1}\right)$. Mass spectra were obtained from the California Institute of Technology Mass Spectroscopy Facility. High performance liquid chromatography (HPLC) was performed on a Hewlett-Packard 1100 Series chromatograph using Daicel Chiracel AD, OD-H, and Regis Whelk-O 2 columns $(25 \mathrm{~cm})$ and equivalent guard columns $(5 \mathrm{~cm})$. Analytical supercritical fluid chromatography (SFC) was performed on a Berger Instruments SFC with built-in photometric detector $(\lambda=214 \mathrm{~nm})$ using Daicel Chiracel OD-H, and AD-H columns $(25 \mathrm{~cm})$ as noted. Optical rotations were measured on a Jasco P-1010 
polarimeter, and $[\alpha]_{D}^{25}$ values are reported in $10^{-1} \mathrm{dg} \mathrm{cm}^{2} \mathrm{~g}^{-1}$; concentration (c) is in $\mathrm{g} / 100$ $\mathrm{mL}$.

General Procedure for the Synthesis of Silyloxycarbamate Nucleophiles: A $100 \mathrm{~mL}$ round-bottom flask charged with hydroxycarbamate (10 mmol, 1.0 equiv) in $\mathrm{CH}_{2} \mathrm{Cl}_{2}$ (50 $\mathrm{mL})$ and triethylamine (1.1 equiv) was cooled to $0{ }^{\circ} \mathrm{C}$ and $\mathrm{TBSCl}$ or TBDPSCl (10 mmol) was added. The reaction was allowed to warm to ambient temperature and stirred for 12 hours. Upon completion, the reaction was poured into a $250 \mathrm{~mL}$ separatory funnel and washed with $\mathrm{H}_{2} \mathrm{O}$, brine, and then dried over $\mathrm{MgSO}_{4}$ and concentrated in vacuo. Purification by silica gel chromatography of the residue afforded the desired silyloxycarbamate.

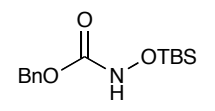

Benzyl-(tert-butyldimethylsilyloxy)carbamate: Prepared according to general procedure using benzyl- $N$-hydroxycarbamate $(1.67 \mathrm{~g}, 10 \mathrm{mmol})$, triethylamine $(1.56 \mathrm{~mL}$, $11 \mathrm{mmol})$, and TBSCl (1.50 g, $10.0 \mathrm{mmol})$. Purification by silica gel chromatography ( $10 \% \mathrm{Et}_{2} \mathrm{O} /$ pentane) provided the title compound as a low melting solid $(2.53 \mathrm{~g}, 90 \%$ yield). IR (film) 3275, 2930, 1730, 1706, 1498, 1472, $1253 \mathrm{~cm}^{-1} ;{ }^{1} \mathrm{H}$ NMR (300 MHz, $\left.\mathrm{CDCl}_{3}\right) \delta 0.08\left(\mathrm{~s}, 6 \mathrm{H}, \mathrm{SiCH}_{3}\right), 0.92$ (s, 9H, SiC $\left.\left(\mathrm{CH}_{3}\right)_{3}\right), 5.18$ (s, 2H, PhCH $)_{2}, 6.95$ (s, 1H, NH), 7.39 (s, 5H, PhH) ppm; ${ }^{13} \mathrm{C} \mathrm{NMR}\left(75 \mathrm{MHz}, \mathrm{CDCl}_{3}\right) \delta-5.8,18.0,25.8,67.6,128.3$, 128.4, 128.5, 135.6, 158.6 ppm ; HRMS (FAB+) exact mass calculated for $\left(\mathrm{C}_{14} \mathrm{H}_{23} \mathrm{NO}_{3} \mathrm{Si}\right)$ requires $m / z[\mathrm{M}+\mathrm{H}] 282.1526$, found $m / z 282.1518$.

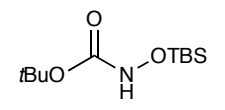

tert-Butyl-(tert-butyldimethylsilyloxy)carbamate: Prepared according to general procedure using tert-Butyl- $N$-hydroxycarbamate $(1.33 \mathrm{~g}, 10 \mathrm{mmol})$, triethylamine (1.56 $\mathrm{mL}, 11 \mathrm{mmol})$, and $\mathrm{TBSCl}(1.50 \mathrm{~g}, 10.0 \mathrm{mmol})$. Purification by silica gel chromatography $\left(10 \% \mathrm{Et}_{2} \mathrm{O} /\right.$ pentane) provided the title compound as a low melting solid (2.27 g, 92\% yield). IR (film) 3292, 2931, 1757, 1694, 1473, 1463, 1393, 1368, 1251, $1172 \mathrm{~cm}^{-1} ;{ }^{1} \mathrm{H}$ NMR $\left(300 \mathrm{MHz}, \mathrm{CDCl}_{3}\right) \delta 0.17\left(\mathrm{~s}, 6 \mathrm{H}, \mathrm{SiCH}_{3}\right), 0.95\left(\mathrm{~s}, 9 \mathrm{H}, \operatorname{SiC}\left(\mathrm{CH}_{3}\right)_{3}\right)$, $1.48\left(\mathrm{~s}, 9 \mathrm{H}, \mathrm{OC}\left(\mathrm{CH}_{3}\right)_{3}\right), 6.67(\mathrm{~s}, 1 \mathrm{H}, \mathrm{NH}) \mathrm{ppm} ;{ }^{13} \mathrm{C} \mathrm{NMR}\left(75 \mathrm{MHz}, \mathrm{CDCl}_{3}\right) \delta-5.8,18.0$, 
25.8, 28.1, 81.5, $157.9 \mathrm{ppm}$; HRMS (FAB+) exact mass calculated for $\left(\mathrm{C}_{11} \mathrm{H}_{25} \mathrm{NO}_{3} \mathrm{Si}\right)$ requires $m / z[\mathrm{M}+\mathrm{H}] 248.1682$, found $m / z$ 248.1679.

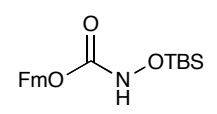

9-Fluorenylmethyl-(tert-butyldimethylsilyloxy)carbamate: Prepared according to general procedure using 9-fluorenylmethyl- $N$-hydroxycarbamate $(2.55 \mathrm{~g}, 10 \mathrm{mmol})$, triethylamine $(1.56 \mathrm{~mL}, 11 \mathrm{mmol})$, and TBSCl $(1.50 \mathrm{~g}, 10.0 \mathrm{mmol})$. Purification by silica gel chromatography $\left(20 \% \mathrm{Et}_{2} \mathrm{O} /\right.$ pentane) provided the title compound as a low melting solid (3.14 g, 85\% yield). IR (film) 3435, 2930, 2856, 1647, 1475, 1450, $1252 \mathrm{~cm}^{-1} ;{ }^{1} \mathrm{H}$

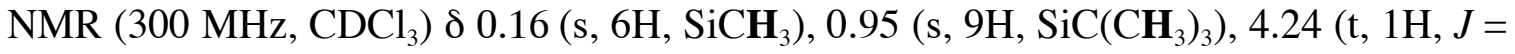
$6.9 \mathrm{~Hz}, \mathrm{CH}), 4.50$ (d, 2H, J = 6.9 Hz, CH$)_{2}, 6.90$ (s, 1H, NH), 7.31-7.43 (m, 4H, ArH), $7.61(\mathrm{~d}, 2 \mathrm{H}, J=7.5 \mathrm{~Hz}, \operatorname{ArH}), 7.76(\mathrm{~d}, 2 \mathrm{H}, J=7.5 \mathrm{~Hz}, \mathrm{ArH}) \mathrm{ppm} ;{ }^{13} \mathrm{C} \mathrm{NMR}(75 \mathrm{MHz}$, $\left.\mathrm{CDCl}_{3}\right) \delta-5.8,18.0,25.9,47.0,67.5,120.0,125.0,127.1,127.8,141.3,143.5,158.7 \mathrm{ppm}$ ; HRMS (FAB+) exact mass calculated for $\left(\mathrm{C}_{21} \mathrm{H}_{27} \mathrm{NO}_{3} \mathrm{Si}\right)$ requires $\mathrm{m} / z[\mathrm{M}+\mathrm{H}] 370.1838$, found $m / z 370.1841$.

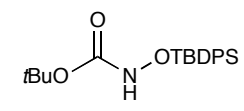

tert-Butyl-(tert-butyldiphenylsilyloxy)carbamate: Prepared according to general procedure using tert-butyl- $N$-hydroxycarbamate $(1.33 \mathrm{~g}, 10 \mathrm{mmol})$, triethylamine (1.56 $\mathrm{mL}, 11 \mathrm{mmol})$, and TBDPSCl $(2.75 \mathrm{~g}, 10.0 \mathrm{mmol})$. Purification by silica gel chromatography (20\% $\mathrm{Et}_{2} \mathrm{O} /$ pentane) provided the title compound as a solid $(3.27 \mathrm{~g}, 88 \%$ yield). IR (film) 3436, 2859, 1757, 1687, 1590, 1473, 1462, 1428, $1247 \mathrm{~cm}^{-1}$; ${ }^{1} \mathrm{H}$ NMR $\left(300 \mathrm{MHz}, \mathrm{CDCl}_{3}\right) \delta 1.14$ (s, 9H, $\left.\mathrm{SiC}\left(\mathrm{CH}_{3}\right)_{3}\right), 1.38$ (s, 9H, OC(CH$\left.)_{3}\right), 6.70$ (s, 1H, NH), 7.30-7.45 (m, 6H, PhH), 7.72-7.76 (m, 4H, PhH) ppm; $\left.{ }^{13} \mathrm{C} \mathrm{NMR} \mathrm{(75} \mathrm{MHz,} \mathrm{CDCl}_{3}\right) \delta 19$, 26.9, 28.0, 81.6, 127.7, 130.0, 132.0, 133.0, 135.8, 157.2 ppm ; HRMS (FAB+) exact mass calculated for $\left(\mathrm{C}_{21} \mathrm{H}_{29} \mathrm{NO}_{3} \mathrm{Si}\right)$ requires $m / z[\mathrm{M}+\mathrm{H}] 372.1995$, found $\mathrm{m} / z 372.1998$.

General Procedure for the Conjugate Amination of Aldehydes: A 4-dram vial 
equipped with a magnetic stir bar was charged with the $p$ TSA or TFA salt of $(2 R, 5 R)-2-$ tert-butyl-5-benzyl-3-methylimidazolidin-4-one $(2)(0.05 \mathrm{mmol})$ and the appropriate enal (0.75 mmol) in $\mathrm{CHCl}_{3}(0.75 \mathrm{~mL})$ was cooled to $-20{ }^{\circ} \mathrm{C}$. The silyloxycarbamate nucleophile $(0.25 \mathrm{mmol})$ was then added in one portion and the reaction mixture was stirred at $-20^{\circ} \mathrm{C}$. Upon completion of the reaction as determined by TLC analysis (12-36 hours), the products were isolated as described below.

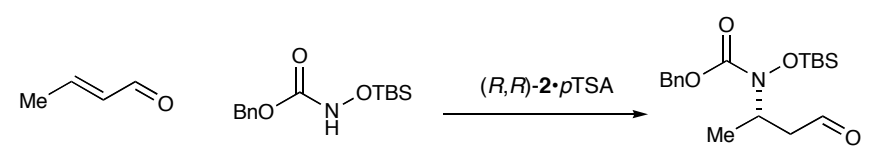

$N$-tert-butyldimethylsilyloxy[benzyl-(S)-1-formylpropan-2-ylcarbamate]: Table 2, entry 1. Prepared according to the general procedure using catalyst $(R, R)-2 \cdot p$ TSA $(21$ $\mathrm{mg}, 0.05 \mathrm{mmol}, 0.2$ equiv), crotonaldehyde $(64 \mu \mathrm{L}, 0.75 \mathrm{mmol})$ in $\mathrm{CHCl}_{3}(0.75 \mathrm{~mL})$ at $-20{ }^{\circ} \mathrm{C}$. Benzyl-(tert-butyldimethylsilyloxy)carbamate $(70 \mathrm{mg}, 0.25 \mathrm{mmol})$ was added in one portion and the reaction was maintained at $-20{ }^{\circ} \mathrm{C}$ for 24 hours. The reaction mixture was then filtered through a plug of silica, eluted with $\mathrm{Et}_{2} \mathrm{O}$ then concentrated in vacuo. Purification by silica gel chromatography ( 5 to $10 \% \mathrm{Et}_{2} \mathrm{O} /$ pentane gradient) provided the title compound as clear oil (81 mg, 92\% yield, 92\% ee). IR (neat) 3445, 2886, 2856, 1709, 1588, 1498, 1472, $1462 \mathrm{~cm}^{-1} ;{ }^{1} \mathrm{H}$ NMR (300 MHz, $\left.\mathrm{CDCl}_{3}\right) \delta 0.09\left(\mathrm{~s}, 3 \mathrm{H}, \mathrm{SiCH}_{3}\right)$, 0.10 (s, 3H, $\left.\mathrm{SiCH}_{3}\right), 0.91$ (s, 9H, $\left.\mathrm{SiC}\left(\mathrm{CH}_{3}\right)_{3}\right), 2.60$ (d, 3H, J = 6.8 Hz, $\left.\mathrm{CHCH}_{3}\right), 2.60$ (dd, $1 \mathrm{H}, J_{1}=6.5$ and $\left.J_{2}=17.0 \mathrm{~Hz}, \mathrm{HCHCHO}\right), 2.82\left(\mathrm{dd}, 1 \mathrm{H}, J_{l}=7.0\right.$ and $J_{2}=17.0 \mathrm{~Hz}$, HCHCHO), 4.51 (m, 1H, NCH), 5.16 (s, 2H, $\left.\mathrm{PhCH}_{2}\right), 7.36$ (m, 5H, PhH), 9.75 (t, J = 1.8 $\mathrm{Hz}, \mathrm{CHO}) \mathrm{ppm} ;{ }^{13} \mathrm{C} \mathrm{NMR}\left(75 \mathrm{MHz}, \mathrm{CDCl}_{3}\right) \delta-4.3,-4.2,18.0,18.5,26.2,47.8,54.0$, 68.3, 128.6, 128.7, 135.9, 159.3, 200.0 ppm ; HRMS (FAB+) exact mass calculated for $\left(\mathrm{C}_{18} \mathrm{H}_{29} \mathrm{NO}_{4} \mathrm{Si}\right)$ requires $m / z[\mathrm{M}+\mathrm{H}] 352.1944$, found $m / z$ 352.1962. $[\alpha]_{D}^{25}=+2.3^{\circ}(\mathrm{c}=1.1$, $\mathrm{CHCl}_{3}$ ). The enantiomeric excess was determined by HPLC analysis of the corresponding alcohol (after treatment with $\mathrm{NaBH}_{4}$ ) using a Regis Technology Whelk-O 2 column (5\% $i \operatorname{PrOH} /$ hexanes, $1.0 \mathrm{~mL} / \mathrm{min}$ ); major enantiomer $\mathrm{t}_{\mathrm{r}}=16 \mathrm{~min}$ and minor enantiomer $\mathrm{t}_{\mathrm{r}}=19$ $\min$. 


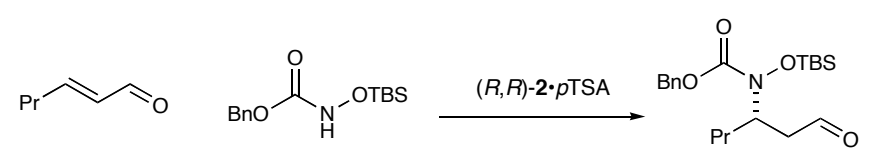

$N$-tert-butyldimethylsilyloxy[benzyl-(S)-1-formylpentan-2-ylcarbamate]: Table 2, entry 2. Prepared according to the general procedure using catalyst $(R, R)-2 \cdot p$ TSA $(21$ $\mathrm{mg}, 0.05 \mathrm{mmol}, 0.2$ equiv), 2-hexenal $(87 \mu \mathrm{L}, 0.75 \mathrm{mmol})$ in $\mathrm{CHCl}_{3}(0.75 \mathrm{~mL})$ at $-20{ }^{\circ} \mathrm{C}$. Benzyl-(tert-butyldimethylsilyloxy)carbamate $(70 \mathrm{mg}, 0.25 \mathrm{mmol})$ was added in one portion and the reaction was maintained $-20^{\circ} \mathrm{C}$ for 24 hours. The reaction mixture was then filtered through a plug of silica, eluted with $\mathrm{Et}_{2} \mathrm{O}$ then concentrated in vacuo. Purification by silica gel chromatography ( 5 to $10 \% \mathrm{Et}_{2} \mathrm{O} /$ pentane gradient) provided the title compound as clear oil (73 mg, 77\% yield, 95\% ee). IR (neat) 3439, 2859, 2722, 1727, 1587, 1498, 1471, $1463 \mathrm{~cm}^{-1} ;{ }^{1} \mathrm{H}$ NMR (300 MHz, $\left.\mathrm{CDCl}_{3}\right) \delta 0.10\left(\mathrm{~s}, 6 \mathrm{H}, \mathrm{SiCH}_{3}\right)$, 0.90 (s, 9H, $\left.\mathrm{SiC}\left(\mathrm{CH}_{3}\right)_{3}\right), 0.80-1.00$ (overlapped t, 3H, $\left.\mathrm{CH}_{2} \mathrm{CH}_{3}\right), 1.38(\mathrm{~m}, 3 \mathrm{H}$, $\left.\mathrm{HCHCH}_{2} \mathrm{CH}_{3}\right), 1.74\left(\mathrm{~m}, 1 \mathrm{H}, \mathbf{H C H C H} \mathrm{CH}_{3}\right), 2.52\left(\mathrm{ddd}, 1 \mathrm{H}, J_{1}=1.9, J_{2}=5.6\right.$ and $J_{3}=$ $16.7 \mathrm{~Hz}, \mathrm{HCHCHO}$ ), 2.80 (ddd, $1 \mathrm{H}, J_{1}=2.1, J_{2}=7.5$ and $\left.J_{3}=16.7 \mathrm{~Hz}, \mathbf{H C H C H O}\right), 4.46$ (m, 1H, NCH), 5.17 (s, 2H, PhCH $), 7.36$ (m, 5H, PhH), 9.76 (t, 1H, J=1.8 Hz, CHO) ppm; ${ }^{13} \mathrm{C} \mathrm{NMR}\left(75 \mathrm{MHz}, \mathrm{CDCl}_{3}\right) \delta-4.5,13.7,18.3,19.5,25.9,34.4,46.4,57.6,68.0$, 128.3, 128.4, 128.5, 135.7, 159.0, 200.0 ppm ; HRMS (EI) exact mass calculated for $\left(\mathrm{C}_{20} \mathrm{H}_{33} \mathrm{NO}_{4} \mathrm{Si}\right)$ requires $m / z[\mathrm{M}+\mathrm{H}] 380.2248$, found $m / z$ 380.2248. $[\alpha]_{D}^{25}=-4.4^{\mathrm{o}}(\mathrm{c}=1.2$, $\mathrm{CHCl}_{3}$ ). The enantiomeric excess was determined by HPLC analysis of the corresponding alcohol (after treatment with $\mathrm{NaBH}_{4}$ ) using a Chiralcel OD-H column (2\% $i \operatorname{PrOH} /$ hexanes, $1 \mathrm{~mL} / \mathrm{min}$ ); major enantiomer $\mathrm{t}_{\mathrm{r}}=20.1$ min and minor enantiomer $\mathrm{t}_{\mathrm{r}}=$ $23.8 \mathrm{~min}$.

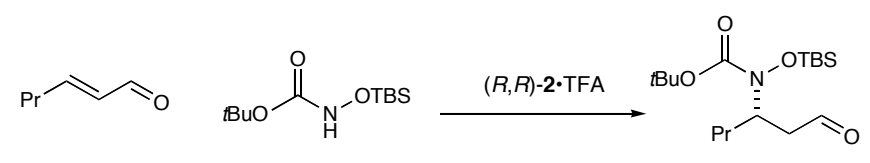

$N$-tert-butyldimethylsilyloxy[tert-butyl-(S)-1-formylpentan-2-ylcarbamate]: Table 2, entry 3. Prepared according to the general procedure using catalyst $(R, R)-2 \cdot \mathrm{TFA}(18 \mathrm{mg}$, $0.05 \mathrm{mmol}, 0.2$ equiv), 2-hexenal $(87 \mu \mathrm{L}, 0.75 \mathrm{mmol})$ in $\mathrm{CHCl}_{3}(0.75 \mathrm{~mL})$ at $-20{ }^{\circ} \mathrm{C}$. tert- 
Butyl-(tert-butyldimethylsilyloxy)carbamate $(62 \mathrm{mg}, 0.25 \mathrm{mmol})$ was added in one portion and the reaction was maintained $-20{ }^{\circ} \mathrm{C}$ for 24 hours. The reaction mixture was then filtered through a plug of silica, eluted with $\mathrm{Et}_{2} \mathrm{O}$ then concentrated in vacuo. Purification by silica gel chromatography ( 5 to $10 \% \mathrm{Et}_{2} \mathrm{O}$ /pentane gradient) provided the title compound as clear oil (80.5 mg, 85\% yield, 92\% ee). IR (neat) 3438, 2898, 2859, 2720, 1728, 1473, $1463 \mathrm{~cm}^{-1}$; ${ }^{1} \mathrm{H}$ NMR (300 MHz, CDCl $) \delta 0.15$ (s, 6H, SiCH $), 0.94$ (s, 9H, $\left.\mathrm{SiC}\left(\mathrm{CH}_{3}\right)_{3}\right), 1.10-1.60\left(\mathrm{~m}, 6 \mathrm{H}, \mathrm{HCHCH}_{2} \mathrm{CH}_{3}\right), 1.47\left(\mathrm{~s}, 9 \mathrm{H}, \mathrm{OC}\left(\mathrm{CH}_{3}\right)_{3}\right), 1.76(\mathrm{~m}, 1 \mathrm{H}$, $\left.\mathbf{H C H C H}_{2} \mathrm{CH}_{3}\right), 2.52\left(\mathrm{dd}, 1 \mathrm{H}, J_{1}=5.7\right.$ and $\left.J_{2}=16.5 \mathrm{~Hz}, \mathrm{HCHCHO}\right), 2.77\left(\mathrm{dd}, 1 \mathrm{H}, J_{1}=\right.$ 7.5 and $\left.J_{2}=16.7 \mathrm{~Hz}, \mathbf{H C H C H O}\right), 4.33$ (m, 1H, NCH), 9.77 (s, 1H, CHO) ppm; ${ }^{13} \mathrm{CNMR}$ $\left(75 \mathrm{MHz}, \mathrm{CDCl}_{3}\right) \delta$-4.42, 13.8, 18.2, 19.6, 26.0, 28.2 34.7, 46.6, 58.1, 81.7, 158.0, 200.9 ppm ; HRMS (EI) exact mass calculated for $\left(\mathrm{C}_{17} \mathrm{H}_{35} \mathrm{NO}_{4} \mathrm{Si}\right)$ requires $m / z[\mathrm{M}+\mathrm{H}]$ 346.2410, found $m / z$ 346.2414. $[\alpha]_{D}^{25}=-1.52^{\circ}\left(\mathrm{c}=0.5, \mathrm{CHCl}_{3}\right)$. The enantiomeric excess was determined by HPLC analysis of the isoxazolidine benzoyl acetal derivative (by treatment with TBAF, then benzoyl chloride and TEA) using Chiralcel OD-H column $(2 \% \mathrm{EtOH} /$ hexanes, $1 \mathrm{~mL} / \mathrm{min})$; major enantiomer $\mathrm{t}_{\mathrm{r}}=7.8 \mathrm{~min}$ and minor enantiomer $\mathrm{t}_{\mathrm{r}}=$ $12.8 \mathrm{~min}$.

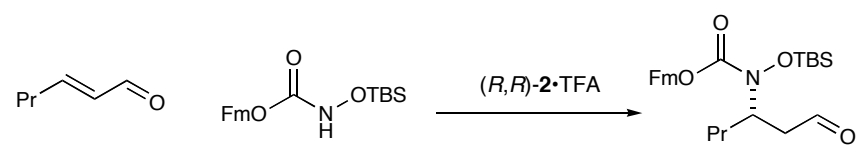

$N$-tert-butyldimethylsilyloxy [(fluoren-9-yl)methyl (S)-1-formylpentan-2ylcarbamate]; Table 2, entry 4. Prepared according to the general procedure using catalyst $(R, R)-2 \cdot p$ TSA ( $21 \mathrm{mg}, 0.05 \mathrm{mmol}, 0.2$ equiv), 2-hexenal ( $87 \mu \mathrm{L}, 0.75 \mathrm{mmol})$ in $\mathrm{CHCl}_{3}(0.75 \mathrm{~mL})$ at $-20{ }^{\circ} \mathrm{C}$. 9-Fluorenylmethyl(tert-butyldimethylsilyloxy)carbamate (92 $\mathrm{mg}, 0.25 \mathrm{mmol}$ ) was added in one portion and the reaction was maintained $-20{ }^{\circ} \mathrm{C}$ for 36 hours. The reaction mixture was then filtered through a plug of silica, eluted with $\mathrm{Et}_{2} \mathrm{O}$ then concentrated in vacuo. Purification by silica gel chromatography (5 to $10 \%$ EtOAc/hexanes gradient) provided the title compound as a clear oil (92 mg, 78\% yield, $89 \%$ ee). IR (neat) 3433, 2858, 2723, 1727, 1471, 1463, 1450, 1390, 1299, 1252, 1200 $\mathrm{cm}^{-1} ;{ }^{1} \mathrm{H}$ NMR $\left(300 \mathrm{MHz}, \mathrm{CDCl}_{3}\right) \delta 0.10\left(\mathrm{~s}, 6 \mathrm{H}, \mathrm{Si}\left(\mathrm{CH}_{3}\right)_{2}\right), 0.83(\mathrm{t}, 3 \mathrm{H}, J=13.0 \mathrm{~Hz}$, $\left.\mathrm{CH}_{3}\right), 0.91\left(\mathrm{~s}, 9 \mathrm{H}, \mathrm{SiC}\left(\mathrm{CH}_{3}\right)_{3}\right), 1.26\left(\mathrm{~m}, 3 \mathrm{H}, \mathrm{HCHCH}_{2} \mathrm{CH}_{3}\right), 1.60\left(\mathrm{~m}, 1 \mathrm{H}, \mathbf{H C H C H} \mathrm{CH}_{3}\right)$, $2.34\left(\mathrm{ddd}, 1 \mathrm{H}, J_{1}=1.9, J_{2}=6.0, J_{3}=17.0 \mathrm{~Hz}, \mathrm{HCHCHO}\right), 2.59\left(\mathrm{ddd}, 1 \mathrm{H}, J_{1}=1.9, J_{2}=\right.$ 
7.2, $\left.J_{3}=17.0 \mathrm{~Hz}, \mathbf{H C H C H O}\right), 4.18(\mathrm{~m}, 1 \mathrm{H}, \mathrm{NCH}), 4.25$ (t, 1H, $\left.J=6.0 \mathrm{~Hz}, \mathrm{CH}\right), 4.59$ (m, 2H, $\left.\mathrm{CH}_{2}\right), 7.28-7.42(\mathrm{~m}, 6 \mathrm{H}, \operatorname{ArH}), 7.61(\mathrm{~d}, 1 \mathrm{H}, J=8.0 \mathrm{~Hz}, \operatorname{ArH}), 7.77$ (d, 1H, $J=8.0$ $\mathrm{Hz}, \operatorname{ArH}), 9.58(\mathrm{t}, 1 \mathrm{H}, J=1.7 \mathrm{~Hz}, \mathrm{CHO}) \mathrm{ppm} ;{ }^{13} \mathrm{C} \mathrm{NMR}\left(75 \mathrm{MHz}, \mathrm{CDCl}_{3}\right) \delta-4.6,13.7$, $18.3,19.5,26.0,34.3,46.3,47.1,58.2,67.3,120.0,124.9,127.1,127.7,141.4,143.6$, 159.0, $200.0 \mathrm{ppm}$; HRMS (EI) exact mass calculated for $\left(\mathrm{C}_{27} \mathrm{H}_{37} \mathrm{NO}_{4} \mathrm{Si}\right)$ requires $\mathrm{m} / \mathrm{z}$ $[\mathrm{M}+\mathrm{H}]$ 468.2586, found $m / z$ 468.2570. $[\alpha]_{D}^{25}=-3.17^{\circ}\left(\mathrm{c}=0.66, \mathrm{CHCl}_{3}\right)$. The enantiomeric ratio was determined by SFC analysis of the corresponding alcohol (after treatment with $\mathrm{NaBH}_{4}$ ) using Chiralcel OD-H column (5 to $50 \%$ gradient $\mathrm{MeOH} / \mathrm{CO}_{2}, 4$ $\mathrm{mL} / \mathrm{min}$ ); minor enantiomer $\mathrm{t}_{\mathrm{r}}=4.22 \mathrm{~min}$ and major enantiomer $\mathrm{t}_{\mathrm{r}}=4.55 \mathrm{~min}$.

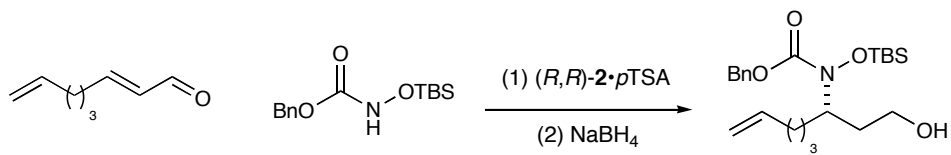

$N$-tert-butyldimethylsilyloxy[benzyl-(S)-hydroxyoct-7-en-3-ylcarbamate]: Table 2, entry 5. Prepared according to the general procedure using catalyst $(R, R)-2 \cdot p$ TSA $(21$ mg, 0.05 mmol, 0.2 equiv), 2,7-octadienal (93 mg, $0.75 \mathrm{mmol})$ in $\mathrm{CHCl}_{3}(0.75 \mathrm{~mL})$ at -20 ${ }^{\circ} \mathrm{C}$. Benzyl-(tert-butyldimethylsilyloxy)carbamate nucleophile $(70 \mathrm{mg}, 0.25 \mathrm{mmol})$ was added in one portion and the reaction was maintained $-20{ }^{\circ} \mathrm{C}$ for 36 hours. Upon completion, $\mathrm{MeOH}(100 \mu \mathrm{L})$ was added to the reaction mixture and warmed to $0{ }^{\circ} \mathrm{C}$ followed by the addition of $\mathrm{NaBH}_{4}(38 \mathrm{mg}, 1 \mathrm{mmol})$. After 1 hour, the excess $\mathrm{NaBH}_{4}$ was quenched by the addition of aqueous $1.0 \mathrm{M} \mathrm{NaHSO}_{4}$ then extracted with $\mathrm{Et}_{2} \mathrm{O}(3 \times 10$ $\mathrm{mL}$ ). The combined organic layer was dried over $\mathrm{MgSO}_{4}$ and concentrated in vacuo. Silica gel chromatography $\left(1 \% \mathrm{MeOH}\right.$ in $\left.\mathrm{CH}_{2} \mathrm{Cl}_{2}\right)$ of the resultant residue afforded the title compound as clear oil (88 mg, 87\% yield, 96\% ee). IR (neat) 3443, 1706, 1641, 1587, 1498, 1472, 1461, $1391 \mathrm{~cm}^{-1}$; ${ }^{1} \mathrm{H}$ NMR (300 MHz, $\left.\mathrm{CDCl}_{3}\right) \delta 0.10\left(\mathrm{~s}, 6 \mathrm{H}, \mathrm{Si}\left(\mathrm{CH}_{3}\right)_{2}\right)$, 0.89 (s, 9H, $\left.\mathrm{SiC}\left(\mathrm{CH}_{3}\right)_{3}\right), 1.31-1.48\left(\mathrm{~m}, 3 \mathrm{H}, \mathrm{HCHCH}_{2} \mathrm{CH}_{2}\right), 1.60-1.95$ (m, 4H, $\left.\mathbf{C H}_{2}\right), 2.04$ $\left(\mathrm{m}, 2 \mathrm{H}, \mathrm{CH}_{2} \mathrm{CH}=\mathrm{CH}_{2}\right), 3.59\left(\mathrm{~m}, 2 \mathrm{H}, \mathrm{CH}_{2} \mathrm{OH}\right), 4.08(\mathrm{~m}, 1 \mathrm{H}, \mathrm{NCH}), 4.91-5.01(\mathrm{~m}, 2 \mathrm{H}$, $\left.\mathrm{HC}=\mathrm{CH}_{2}\right), 5.15\left(\mathrm{~s}, 2 \mathrm{H}, \mathrm{PhCH}_{2}\right)$, 5.65-5.85 (m, $\left.1 \mathrm{H}, \mathrm{CH}_{2} \mathbf{H C}=\mathrm{CH}_{2}\right), 7.31-7.78(\mathrm{~m}, 5 \mathrm{H}$, $\mathrm{PhH}) \mathrm{ppm} ;{ }^{13} \mathrm{C} \mathrm{NMR}\left(75 \mathrm{MHz}, \mathrm{CDCl}_{3}\right) \delta$ 0.06, 0.13, 6.2, 22.8, 30.4, 36.7, 37.9, 39.3, 63.9, 72.5, 89.2, 119.2, 132.8, 132.9, 133.0, 133.1, 140.1, 142.9, 164.0 ppm ; HRMS (EI) exact mass calculated for $\left(\mathrm{C}_{22} \mathrm{H}_{37} \mathrm{NO}_{4} \mathrm{Si}\right)$ requires $\mathrm{m} / \mathrm{z}[\mathrm{M}+\mathrm{H}] 408.2570$, found $\mathrm{m} / \mathrm{z}$ 
408.2570. $[\alpha]_{D}^{25}=+1.0^{\circ}\left(\mathrm{c}=1.0, \mathrm{CHCl}_{3}\right)$. The enantiomeric excess was determined by HPLC analysis of the alcohol using Chiralcel OD-H column ( $2 \% \mathrm{iPrOH} /$ hexanes, 0.75 $\mathrm{mL} / \mathrm{min}$ ); major enantiomer $\mathrm{t}_{\mathrm{r}}=20.9 \mathrm{~min}$ and minor enantiomer $\mathrm{t}_{\mathrm{r}}=25 \mathrm{~min}$.

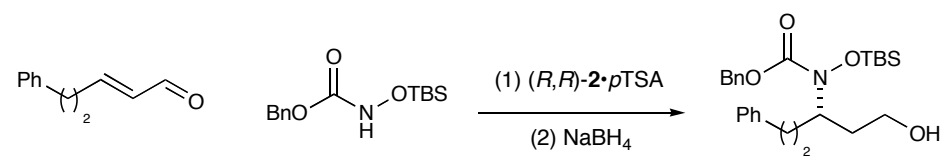

\section{$N$-tert-butyldimethylsilyloxy[benzyl-(S)-5-hydroxyphenylpenta-3-ylcarbamate]:}

Table 2, entry 6. Prepared according to the general procedure using catalyst $(R, R)$ 2.pTSA (21 mg, $0.05 \mathrm{mmol}, 0.2$ equiv), 5-phenylpent-2-enal (120 mg, $0.75 \mathrm{mmol}$ ) in $\mathrm{CHCl}_{3}(0.75 \mathrm{~mL})$ at $-20{ }^{\circ} \mathrm{C}$. Benzyl-(tert-butyldimethylsilyloxy)carbamate (70 mg, 0.25 mmol) was added in one portion and the reaction was maintained $-20{ }^{\circ} \mathrm{C}$ for 36 hours. Upon completion, $\mathrm{MeOH}(100 \mu \mathrm{L})$ was added to the reaction mixture and warmed to 0 ${ }^{\circ} \mathrm{C}$ followed by addition of $\mathrm{NaBH}_{4}(38 \mathrm{mg}, 1 \mathrm{mmol})$. After 1 hour, excess $\mathrm{NaBH}_{4}$ was quenched by addition of aqueous $1.0 \mathrm{M} \mathrm{NaHSO}_{4}$ then extracted with $\mathrm{Et}_{2} \mathrm{O}(3 \times 10 \mathrm{~mL})$. The combined organic layer was dried over $\mathrm{MgSO}_{4}$ and concentrated in vacuo. Purification by silica gel chromatography (20\% EtOAc/hexanes) provided the title compound as clear oil (77 mg, 69\% yield, 90\% ee). IR (film) 3450, 2953, 2930, 1948, 1871, 1805, 1709, 1603, 1587, 1497, 1472, 1455, 1403, 1391, 1362, 1253, $1214 \mathrm{~cm}^{-1} ;{ }^{1} \mathrm{H}$ NMR (300 MHz, $\mathrm{CDCl}_{3}$ ) 80.13 (s, 3H, SiCH $\left._{3}\right), 0.14$ (s, 3H, SiCH $), 0.92$ (s, 9H, $\left.\mathrm{SiC}\left(\mathrm{CH}_{3}\right)_{3}\right), 2.17-1.63\left(\mathrm{~m}, 5 \mathrm{H},\left(\mathrm{CH}_{2}\right)_{2}, \mathbf{O H}\right), 2.76-2.55\left(\mathrm{~m}, 2 \mathrm{H}, \mathrm{CH}_{2}\right), 3.67-3.54(\mathrm{~m}, 2 \mathrm{H}$, $\left.\mathrm{CH}_{2} \mathrm{OH}\right), 4.19-4.09(\mathrm{~m}, 1 \mathrm{H}, \mathrm{NCH}), 5.17\left(\mathrm{~s}, 2 \mathrm{H}, \mathrm{PhCH}_{2} \mathrm{O}\right), 7.37-7.13(\mathrm{~m}, 10 \mathrm{H}, \mathrm{PhH})$ ppm; ${ }^{13} \mathrm{C}$ NMR $\left(75 \mathrm{MHz}, \mathrm{CDCl}_{3}\right) \delta-4.2,-4.1,18.6,26.2,33.2,34.8,35.2,59.6,59.7$, 68.3, 126.1, 128.61, 128.63, 128.7, 128.8, 128.9, 135.9, 141.9, 159.8 ppm ; HRMS (FAB) exact mass calculated for $\left(\mathrm{C}_{25} \mathrm{H}_{37} \mathrm{NO}_{4} \mathrm{Si}\right)$ requires $\mathrm{m} / z$ [M+H] 444.2570, found $\mathrm{m} / \mathrm{z}$ 444.2551. $[\alpha]_{D}^{25}=+0.99\left(\mathrm{c}=0.58, \mathrm{CHCl}_{3}\right)$. The enantiomeric excess was determined by HPLC using a Regis Technology $(R, R)$-Whelk-O 2 column $(5 \% i \mathrm{PrOH} /$ hexanes, 0.75 $\mathrm{mL} / \mathrm{min}$ ); major enantiomer $\mathrm{t}_{\mathrm{r}}=27.7$ min and minor $\mathrm{t}_{\mathrm{r}}=30.6 \mathrm{~min}$.

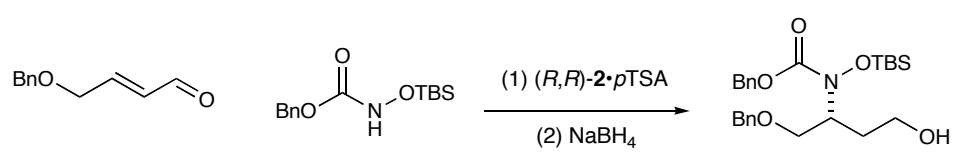




\section{$N$-tert-butyldimethylsilyloxy[benzyl-(S)-(benzyloxy)-4-hydroxybutan-2-}

ylcarbamate]: Table 2, entry 7. Prepared according to the general procedure using catalyst $(R, R)$-2·pTSA ( $21 \mathrm{mg}, 0.05 \mathrm{mmol}, 0.2$ equiv), 4-(benzyloxy)but-2-enal (132 mg, $0.75 \mathrm{mmol})$ in $\mathrm{CHCl}_{3}(0.75 \mathrm{~mL})$ at $-20{ }^{\circ} \mathrm{C}$. Benzyl-(tert-butyldimethylsilyloxy)carbamate (70 $\mathrm{mg}, 0.25 \mathrm{mmol}$ ) was added in one portion and the reaction was maintained $-20{ }^{\circ} \mathrm{C}$ for 36 hours. Upon completion, $\mathrm{MeOH}(100 \mu \mathrm{L})$ was added to the reaction mixture and warmed to $0{ }^{\circ} \mathrm{C}$ followed by the addition of $\mathrm{NaBH}_{4}(38 \mathrm{mg}, 1 \mathrm{mmol})$. After 1 hour, excess $\mathrm{NaBH}_{4}$ was quenched by the addition of aqueous $1.0 \mathrm{M} \mathrm{NaHSO}_{4}$ then extracted with $\mathrm{Et}_{2} \mathrm{O}(3 \times 10 \mathrm{~mL})$. The combined organic layer was dried over $\mathrm{MgSO}_{4}$ and concentrated in vacuo. Purification by silica gel chromatography (20\% EtOAc/hexanes) provided the title compound as clear oil (80 mg, 70\% yield, 96\% ee). IR (film) 3450, 3065, 3032, 2953, 2929, 2884, 2858, 2741, 2712, 1950, 1874, 1808, 1713, 1606, 1587, $1497,1472,1455,1390,1362,1305,1252,1214 \mathrm{~cm}^{-1} ;{ }^{1} \mathrm{H}$ NMR $\left(300 \mathrm{MHz}, \mathrm{CDCl}_{3}\right) \delta$ 0.11 (s, 3H, $\left.\mathbf{S i C H}_{3}\right) 0.12$ (s, 3H, $\left.\mathrm{SiCH}_{3}\right), 0.90$ (s, 9H, $\left.\mathrm{SiC}\left(\mathrm{CH}_{3}\right)_{3}\right), 1.94-1.65$ (m, 2H, $\mathrm{CH}_{2} \mathrm{CH}_{2} \mathrm{OH}$ ), 2.44 (br s, 1H, OH), 3.78-3.45 (m, 4H, BnOCH $\mathbf{C H}_{2} \mathrm{OH}$ ), 4.36-4.27 (m, $1 \mathrm{H}, \mathrm{CHN}), 4.51$ (s, 2H, $\mathrm{PhCH}_{2} \mathrm{O}$ ), 5.13 (s, 2H, $\left.\mathrm{PhCH}_{2} \mathrm{O}\right), 7.35-7.31(\mathrm{~m}, 10 \mathrm{H}, \mathrm{PhH}) ;{ }^{13} \mathrm{C}$ NMR (75 MHz, $\left.\mathrm{CDCl}_{3}\right) \delta$-4.6, -4.2, 18.5, 26.2, 31.9, 59.8, 60.1, 68.3, 70.4, 73.2, 127.8, 127.9, 128.6, 128.7, 128.8, 135.9, 138.3, 160.0 ppm; HRMS (FAB) exact mass calculated for $\left(\mathrm{C}_{25} \mathrm{H}_{37} \mathrm{NO}_{5} \mathrm{Si}\right)$ requires $\mathrm{m} / z[\mathrm{M}+\mathrm{H}] 460.2519$, found $\mathrm{m} / z$ 460.2536. $[\alpha]_{D}^{25}=+7.51^{\circ}(\mathrm{c}$ $\left.=0.50, \mathrm{CHCl}_{3}\right)$. The enantiomeric excess was determined by HPLC using a Regis Technology $(R, R)$ Whelk-O $2(25 \mathrm{~cm} \times 4.6 \mathrm{~mm})$ column $(5 \% i \mathrm{PrOH} /$ hexanes, 1.0 $\mathrm{mL} / \mathrm{min}$ ); major isomer $\mathrm{t}_{\mathrm{r}}=26.6 \mathrm{~min}$ and minor isomer $\mathrm{t}_{\mathrm{r}}=31.8 \mathrm{~min}$.

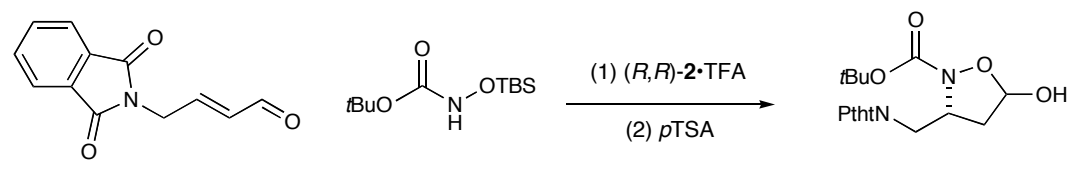

(3S,5S)-tert-Butyl-3-( $N$-methylphthalimido)-5-hydroxyisoxazolidine-2-carboxylate:

Table 2, entry 8. Prepared according to the general procedure using catalyst $(R, R)-2 \cdot$ TFA (18 mg, $0.05 \mathrm{mmol}, 0.2$ equiv), 4-( $N$-methylphthalimido)but-2-enal (161 mg, $0.75 \mathrm{mmol}$ ) in $\mathrm{CHCl}_{3}(0.75 \mathrm{~mL})$ at $-20{ }^{\circ} \mathrm{C}$. tert-Butyl-(tert-butyldimethylsilyloxy)carbamate (62 $\mathrm{mg}$, $0.25 \mathrm{mmol}$ ) was added in one portion and the reaction was maintained $-20{ }^{\circ} \mathrm{C}$ for 36 
hours. The reaction was warmed to ambient temperature followed the addition of acetone- $\mathrm{H}_{2} \mathrm{O}(1 \mathrm{~mL}, 4: 1)$ and $p \mathrm{TSA} \cdot \mathrm{H}_{2} \mathrm{O}(47.5 \mathrm{mg}, 0.25 \mathrm{mmol})$. After 12 hours the reaction was passed through a plug of silica gel, eluted with $\mathrm{Et}_{2} \mathrm{O}$ and concentrated in vacuo. Purification by silica gel chromatography (20 to $30 \% \mathrm{EtOAc/hexanes} \mathrm{gradient)}$ provided the title compound as amorphous solid ( $74 \mathrm{mg}, 85 \%$ yield, $87 \%$ ee). IR (film) 3470, 3362, 1774, 1716, 1614, 1468, 1430, 1396, 1370, $1354 \mathrm{~cm}^{-1}$; ${ }^{1} \mathrm{H}$ NMR (300 MHz, $\left.\mathrm{CDCl}_{3}\right) \delta 1.24\left(\mathrm{~s}, 9 \mathrm{H}, \mathrm{OC}\left(\mathrm{CH}_{3}\right)_{3}\right), 2.05(\mathrm{~m}, 1 \mathrm{H}, \mathrm{HCH}), 2.40\left(\mathrm{dd}, 1 \mathrm{H}, J_{1}=8.4\right.$ and $J_{2}=$

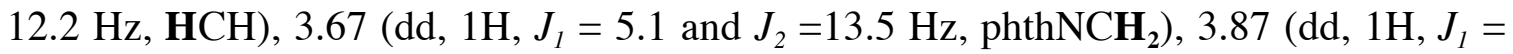
8.4 and $J_{2}=13.5 \mathrm{~Hz}$, phthNCH$\left.)_{2}\right), 4.67(\mathrm{~m}, 1 \mathrm{H}, \mathrm{NCH}), 5.29$ (br s, $\left.1 \mathrm{H}, \mathrm{OH}\right), 5.78(\mathrm{~d}, 1 \mathrm{H}, J$ $=4.8 \mathrm{~Hz}, \mathrm{OCHOH}), 7.69-7.75(\mathrm{~m}, 2 \mathrm{H}, \mathrm{ArH}), 7.82-7.89(\mathrm{~m}, 2 \mathrm{H}, \mathrm{ArH}) \quad \mathrm{ppm} ;{ }^{13} \mathrm{C} \mathrm{NMR}$ $\left(75 \mathrm{MHz}, \mathrm{CDCl}_{3}\right)$ \& 27.9, 39.5, 41.6, 56.5, 82.5, 98.7, 123.4, 132.0, 134.0, 158.4, 168.0 ppm; HRMS (EI) exact mass calculated for $\left(\mathrm{C}_{17} \mathrm{H}_{20} \mathrm{~N}_{2} \mathrm{O}_{6}\right)$ requires $\mathrm{m} / z$ [M+H] 349.1400, found $m / z$ 349.1398. $[\alpha]_{D}^{25}=-60.0^{\circ}\left(\mathrm{c}=0.6, \mathrm{CHCl}_{3}\right)$. The enantiomeric ratio was determined by SFC analysis using a Chiralcel OD-H column (5 to $50 \% \mathrm{MeOH} / \mathrm{CO}_{2}$ gradient, $4 \mathrm{~mL} / \mathrm{min}$ ); major enantiomer $t_{r}=3.1 \mathrm{~min}$ and minor enantiomer $t_{r}=3.45 \mathrm{~min}$.

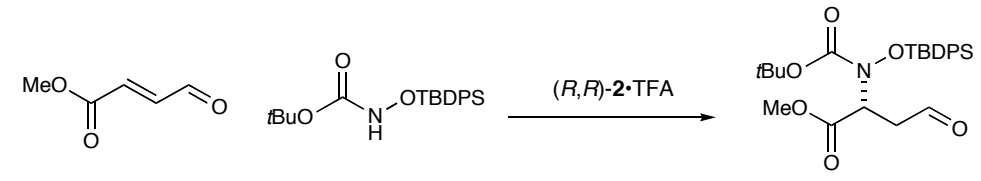

$N$-tert-butyldimethylsilyloxy[tert-butyl-(R)-1-methoxycarbonyl-2-

formylethylcarbamate]: Table 2, entry 9. Prepared by the modification of the general procedure using catalyst $(R, R)$-2.TFA (18 $\mathrm{mg}, 0.05 \mathrm{mmol}, 0.2$ equiv), methyl-3formoylacrylate $(86 \mathrm{mg}, 0.75 \mathrm{mmol})$ in $\mathrm{CHCl}_{3}(0.25 \mathrm{~mL})$ at $4{ }^{\circ} \mathrm{C}$. tert-Butyl-(tertbutyldiphenylsilyloxy)carbamate (93 $\mathrm{mg}, 0.25 \mathrm{mmol}$ ) was added in one portion and the reaction was maintained $4{ }^{\circ} \mathrm{C}$ for 12 hours. The reaction mixture was then filtered through a plug of silica, eluted with $\mathrm{Et}_{2} \mathrm{O}$ then concentrated in vacuo. Purification by silica gel chromatography ( 10 to $20 \%$ of $\mathrm{Et}_{2} \mathrm{O} /$ pentane gradient) provided the title compound as clear oil (95 mg, 78\% yield, 97\% ee). IR (neat) 3421, 2895, 2859, 2727, 1731, 1590, 1568, 1488, 1463, 1474, $1429 \mathrm{~cm}^{-1}$; ${ }^{1} \mathrm{H}$ NMR (300 MHz, $\mathrm{CDCl}_{3}$ ) $\delta 1.14$ (s, $\left.9 \mathrm{H}, \mathrm{SiC}\left(\mathrm{CH}_{3}\right)_{3}\right), 1.22\left(\mathrm{~s}, 9 \mathrm{H}, \mathrm{OC}\left(\mathrm{CH}_{3}\right)_{3}\right), 2.60\left(\mathrm{dd}, 1 \mathrm{H}, J_{1}=5.0\right.$ and $J_{2}=17.8 \mathrm{~Hz}$, $\mathrm{HCHCHO}$ ), $3.12\left(\mathrm{ddd}, 1 \mathrm{H}, J_{l}=1.3, J_{2}=8.3\right.$ and $\left.J_{3}=17.8 \mathrm{~Hz}, \mathbf{H C H C H O}\right), 3.56(\mathrm{~s}, 3 \mathrm{H}$, 
$\left.\mathrm{OCH}_{3}\right), 4.84\left(\mathrm{dd}, 1 \mathrm{H}, J_{l}=6.0\right.$ and $\left.J_{2}=8.2 \mathrm{~Hz}, \mathrm{NCH}\right), 7.33-7.45(\mathrm{~m}, 6 \mathrm{H}, \mathrm{PhH}), 7.66-7.76$ (m, 4H, PhH), 9.55 (s, 1H, CHO) ppm; ${ }^{13} \mathrm{C}$ NMR $\left(75 \mathrm{MHz}, \mathrm{CDCl}_{3}\right.$ ) $\delta$ 19.4, 27.0 27.8, 43.1, 52.3, 60.8, 82.7, 127.5, 127.6, 127.8, 129.6, 130.0, 132.0, 132.4, 136.0, 136.3, 136.4, 158.8, 169.8, $198.3 \mathrm{ppm}$; HRMS (EI) exact mass calculated for $\left(\mathrm{C}_{26} \mathrm{H}_{35} \mathrm{NO}_{6} \mathrm{Si}\right)$ requires $m / z[\mathrm{M}+\mathrm{H}] 486.2312$, found $m / z$ 486.2308. $[\alpha]_{D}^{25}=+22.7^{\circ}\left(\mathrm{c}=0.80, \mathrm{CHCl}_{3}\right)$. The enantiomeric excess was determined by SFC analysis of the aldehyde Chiralcel OD$\mathrm{H}$ column (10\% $\left.\mathrm{AcCN} / \mathrm{CO}_{2}, 4 \mathrm{~mL} / \mathrm{min}\right)$; minor enantiomer $\mathrm{t}_{\mathrm{r}}=1.91 \mathrm{~min}$ and major enantiomer $\mathrm{t}_{\mathrm{r}}=2.14 \mathrm{~min}$.

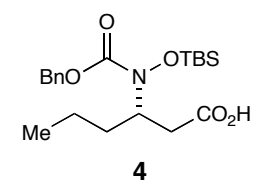

$N$-tert-butyldimethylsilyloxy[benzyl (S)-3-carbamate-hexanoic acid] (4): Prepared according to the general procedure using catalyst $(R, R)-\mathbf{2} \cdot \mathrm{pTSA}(84 \mathrm{mg}, 0.20 \mathrm{mmol}, 0.2$ equiv) in $\mathrm{CHCl}_{3}(3 \mathrm{~mL})$, 2-hexenal $(348 \mu \mathrm{L}, 3 \mathrm{mmol})$ in $\mathrm{CHCl}_{3}(0.75 \mathrm{~mL})$ at $-20{ }^{\circ} \mathrm{C}$. Benzyl-(tert-butyldimethylsilyloxy)carbamate $(281 \mathrm{mg}, 1.0 \mathrm{mmol})$ was added in one portion and the reaction was maintained at $-20{ }^{\circ} \mathrm{C}$ for 24 hours. Upon completion (a small aliquot was removed for ee determination in accord to table 2 , entry 2) the reaction was warmed to $4{ }^{\circ} \mathrm{C}$, isobutene $(1 \mathrm{~mL})$, tert-butanol $(4 \mathrm{~mL}), \mathrm{H}_{2} \mathrm{O}(2 \mathrm{~mL}), \mathrm{KH}_{2} \mathrm{PO}_{4},(544$ $\mathrm{mg}, 4 \mathrm{mmol})$, and $\mathrm{NaClO}_{2}(360 \mathrm{mg}, 4 \mathrm{mmol})$ were added sequentially. The reaction was allowed to stir at this tempearature for 12 hours. The mixture was diluted with $\mathrm{H}_{2} \mathrm{O}$ (10 $\mathrm{mL})$ and the organic layer separated. The aqueous layer was extracted with $\mathrm{CH}_{2} \mathrm{Cl}_{2}(3 \mathrm{X}$ $20 \mathrm{~mL}$ ) and the combined organic layer washed with brine, dried over $\mathrm{MgSO}_{4}$ and concentrated in vacuo. Purification by silica gel chromatography (5 to $10 \% \mathrm{Et}_{2} \mathrm{O} /$ pentane gradient) provided the title compound as clear oil (285 mg, $72 \%$ yield, 92\%ee). IR (neat) 3200, 2859, 1713, 1432, 1391, 1293, 1257, $1089 \mathrm{~cm}^{-1}$; ${ }^{1} \mathrm{H}$ NMR (300 MHz, $\left.\mathrm{CDCl}_{3}\right) \delta 0.11\left(\mathrm{~s}, 6 \mathrm{H}, \mathrm{Si}\left(\mathrm{CH}_{3}\right)_{2}\right), 0.87$ (t, 3H, $\left.J=6 \mathrm{~Hz}, \mathrm{CH}_{3}\right), 0.90$ (s, 9H, $\left.\operatorname{SiC}\left(\mathrm{CH}_{3}\right)_{3}\right)$, 1.26-1.45 (m, $\left.3 \mathrm{H}, \mathbf{H C H C H} \mathbf{C H}_{3}\right), 1.73\left(\mathrm{~m}, 1 \mathrm{H}, \mathbf{H C H C H} \mathrm{CH}_{3}\right), 2.50\left(\mathrm{dd}, 1 \mathrm{H}, J_{1}=6.3\right.$ and $\left.J_{2}=16.0 \mathrm{~Hz}, \mathrm{HCHCO}_{2} \mathrm{H}\right), 2.76\left(\mathrm{dd}, 1 \mathrm{H}, J_{1}=7.2\right.$ and $\left.J_{2}=16.0 \mathrm{~Hz}, \mathbf{H C H C O}_{2} \mathrm{H}\right), 4.37(\mathrm{~m}$, $1 \mathrm{H}, \mathrm{NCH}), 5.15$ (s, 2H, $\left.\mathrm{PhCH}_{2}\right), 7.35(\mathrm{~m}, 5 \mathrm{H}, \mathrm{PhH}) \mathrm{ppm} ;{ }^{13} \mathrm{C} \mathrm{NMR}\left(75 \mathrm{MHz}, \mathrm{CDCl}_{3}\right) \delta$ $-4.7,-4.5,13.7,18.3,19.5,25.9,34.4,37.1,53.4,59.0,67.9,128.2,128.3,128.4,135.8$, 
159.1, 176.9 ppm; HRMS (EI) exact mass calculated for $\left(\mathrm{C}_{20} \mathrm{H}_{33} \mathrm{NO}_{5} \mathrm{Si}\right)$ requires $\mathrm{m} / \mathrm{z}$ $[\mathrm{M}+\mathrm{H}]$ 396.2206, found $m / z$ 396.2201. $[\alpha]_{D}^{25}=-2.23^{\circ}\left(\mathrm{c}=0.64, \mathrm{CHCl}_{3}\right)$.

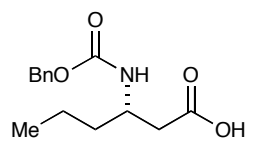

Benzyl (S)-3-carbamate-hexanoic acid: A 4-dram vial charged with 3 (39 mg, 0.1 mmol) in $\mathrm{AcOH}-\mathrm{H}_{2} \mathrm{O}(250 \mu \mathrm{L}, 4: 1)$ was heated to $65^{\circ} \mathrm{C}$, after 15 min $\mathrm{Zn}$ dust (4 X 16.4 $\mathrm{mg}, 1.0 \mathrm{mmol}, 10$ micron) was added in 4 portions at 30 minute intervals. The reaction was maintained at $65{ }^{\circ} \mathrm{C}$ for 6 hours. Upon cooling to ambient temperature, the reaction was diluted with $\mathrm{H}_{2} \mathrm{O}(2 \mathrm{~mL}), 1 \mathrm{M} \mathrm{HCl}(3 \mathrm{~mL})$, and aqueous solution was extracted with EtOAc $(3 \times 10 \mathrm{~mL})$. The combined organic layers was washed successively with $\mathrm{H}_{2} \mathrm{O}$, brine, then dried over $\mathrm{MgSO}_{4}$ and concentrated in vacuo. Purification by silica gel chromatography (20 to $30 \%$ EtOAc/hexanes gradient) provided the title compound as a solid (24.4 mg, 92\% yield). All spectroscopic data matched literature reported values. ${ }^{2}$ IR (film) 3329, 2957, 1693, 1539, 1455, 1303, $1266 \mathrm{~cm}^{-1}$; ${ }^{1} \mathrm{H}$ NMR (300 MHz, $\left.\left.\mathrm{CDCl}_{3}\right) \delta 0.9 \mathfrak{t t}, J=7.0 \mathrm{~Hz}, \mathrm{CH}_{3}\right), 1.35\left(\mathrm{~m}, 2 \mathrm{H}, \mathrm{CH}_{2} \mathrm{CH}_{2} \mathrm{CH}_{3}\right), 1.54(\mathrm{~m}, 2 \mathrm{H}$, $\left.\mathrm{CH}_{2} \mathrm{CH}_{2} \mathrm{CH}_{3}\right), 2.60\left(\mathrm{~m}, 2 \mathrm{H}, \mathrm{CH}_{2} \mathrm{CO}_{2} \mathrm{H}\right), 3.99(\mathrm{~m}, 1 \mathrm{H}, \mathrm{NCH}), 5.09$ (s, 2H, $\left.\mathrm{PhCH}_{2}\right), 5.18$ (br, 1H, NH), 7.35 (m, 5H, PhH) ppm; ${ }^{13} \mathrm{C}$ NMR $\left(75 \mathrm{MHz}, \mathrm{CDCl}_{3}\right) \delta$ 13.7, 19.4, 36.4, 38.7, 47.7, 66.8, 128.1, 128.2, 128.5, 136.4, 155.9, 176.7 ppm; HRMS (EI) exact mass calculated for $\left(\mathrm{C}_{14} \mathrm{H}_{19} \mathrm{NO}_{4}\right)$ requires $\mathrm{m} / \mathrm{z} 265.1314[\mathrm{M}+]$, found $\mathrm{m} / \mathrm{z}$ 265.1311. $[\alpha]_{D}^{25}=$ $-11.45^{\circ}(\mathrm{c}=0.50, \mathrm{MeOH})$. The observed optical rotation was consitent with the $(S)$ configuration for benzyl (S)-3-carbamate-hexanoic acid, based on the previously reported $(R)$-configuration ${ }^{3}$ having the opposite sign of rotation $\left([\alpha]_{D}^{25}=+12.9^{\circ}(\mathrm{c}=0.55\right.$, $\mathrm{MeOH})$.

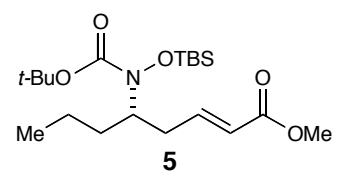

$N$-tert-butyldimethylsilyloxy[tert-butyl-(S)-1-(methoxycarbonyl)hept-1-en-4-

ylcarbamate] (5): Prepared according to the general procedure using catalyst $(R, R)$ - 
2.TFA ( $72 \mathrm{mg}, 0.20 \mathrm{mmol}, 0.2$ equiv), 2-hexenal ( $348 \mu \mathrm{L}, 3 \mathrm{mmol})$ in $\mathrm{CHCl}_{3}(3 \mathrm{~mL})$ at $-20{ }^{\circ} \mathrm{C}$. tert-Butyl-(tert-butyldimethylsilyloxy)carbamate $(247 \mathrm{mg}, 1.0 \mathrm{mmol})$ was added in one portion and the reaction was maintained at $-20{ }^{\circ} \mathrm{C}$ for 24 hours. Upon completion (determined by TLC), methyl (triphenylphosphorylidine) acetate $(1.03 \mathrm{~g}, 3.0 \mathrm{mmol})$ was added and allowed to warm to ambient temperature. After 2 hours, the reaction mixture was concentrated to a viscous oil, and the triphenyphosphine oxide byproduct was precipitated by the addition of $\mathrm{Et}_{2} \mathrm{O}$. The solid was removed by filtration and the organic layer concentrated in vacuo. Purification by silica gel chromatography (2.5 to $10 \%$ $\mathrm{Et}_{2} \mathrm{O} /$ pentane gradient) provided the title compound as clear oil ( $285 \mathrm{mg}, 71 \%$ yield). IR (neat) 2933, 2859, 1729, 1704, 1660, 1472, 1466, 1392, 1362, $1319 \mathrm{~cm}^{-1} ;{ }^{1} \mathrm{H}$ NMR (300 $\left.\mathrm{MHz}, \mathrm{CDCl}_{3}\right) \delta 0.15\left(\mathrm{~s}, 6 \mathrm{H}, \mathrm{Si}\left(\mathrm{CH}_{3}\right)_{2}\right), 0.91\left(\mathrm{t}, 3 \mathrm{H}, J=7.5 \mathrm{~Hz}, \mathrm{CH}_{3}\right), 0.95(\mathrm{~s}, 9 \mathrm{H}$, $\left.\mathrm{SiC}\left(\mathrm{CH}_{3}\right)_{3}\right), 1.3-1.40\left(\mathrm{~m}, 3 \mathrm{H}, \mathrm{HCHCH} \mathrm{CH}_{3}\right), 1.45\left(\mathrm{~s}, 9 \mathrm{H}, \mathrm{O}\left(\mathrm{CCH}_{3}\right)_{3}\right), 1.70(\mathrm{~m}, 1 \mathrm{H}$, $\mathbf{H C H C H}_{2} \mathrm{CH}_{3}$ ), 2.28 (ddd, $1 \mathrm{H}, J_{I}=5.7, J_{2}=12.9$ and $\left.J_{3}=20.4 \mathrm{~Hz}, \mathrm{HCHCCH}=\right), 2.63$ (ddd, $1 \mathrm{H}, J_{1}=7.2, J_{2}=14.4$ and $\left.J_{3}=20.4 \mathrm{~Hz}, \mathbf{H C H C H}=\right), 3.71\left(\mathrm{~s}, 3 \mathrm{H}, \mathrm{CO}_{2} \mathrm{CH}_{3}\right), 3.95$ $(\mathrm{m}, 1 \mathrm{H}, \mathrm{NCH}), 5.88\left(\mathrm{~d}, 1 \mathrm{H}, J=7.2 \mathrm{~Hz}, \mathrm{CH}_{2} \mathrm{CH}=\mathrm{CHCO}_{2} \mathrm{CH}_{3}\right), 6.94\left(\mathrm{dt}, 1 \mathrm{H}, J_{1}=7.2\right.$ and $\left.J_{2}=14.4 \mathrm{~Hz}, \mathrm{CH}_{2} \mathrm{CH}=\mathrm{CH}\right) \mathrm{ppm} ;{ }^{13} \mathrm{C}$ NMR $\left(75 \mathrm{MHz}, \mathrm{CDCl}_{3}\right) \delta-4.3,-4.4,13.8,18.2$, 19.7, 26.0, 28.2, 34.5, 35.3, 51.3, 61.6 81.3, 122.9, 146.3, 158.6, 166.8 ppm ; HRMS $(\mathrm{FAB}+)$ exact mass calculated for $\left(\mathrm{C}_{20} \mathrm{H}_{39} \mathrm{NO}_{5} \mathrm{Si}\right)$ requires $[\mathrm{M}-\mathrm{H}] \mathrm{m} / \mathrm{z} 400.2519$, found $m / z$ 400.2514. $[\alpha]_{D}^{25}=-8.3^{\circ}\left(\mathrm{c}=0.765, \mathrm{CHCl}_{3}\right)$. The enantiomeric excess was determined by HPLC using a Regis Technology $(R, R)$-Whelk-O 2 column $(0.5 \% i \mathrm{PrOH} /$ hexanes, $0.75 \mathrm{~mL} / \mathrm{min}, 215 \mathrm{~nm}$ ); Major enantiomer $\mathrm{t}_{\mathrm{r}}=21.0 \mathrm{~min}$ and $\operatorname{minor} \mathrm{t}_{\mathrm{r}}=22.8 \mathrm{~min}$.

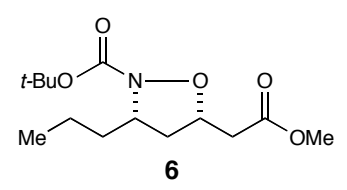

(3S,5S)-tert-Butyl-5-((methoxycarbonyl)methyl)-3-propylisoxazolidine-2-carboxylate (6): A 4 dram screw-cap vial charged with 5 (150 mg, $0.37 \mathrm{mmol})$ in $\mathrm{CH}_{2} \mathrm{Cl}_{2}(2 \mathrm{~mL})$ was added acetic acid $(29 \mu \mathrm{L}, 0.50 \mathrm{mmol})$. The mixture was cooled to $0{ }^{\circ} \mathrm{C}$ and TBAF $(0.5$ $\mathrm{mL}, 0.5 \mathrm{mmol}, 1 \mathrm{M}$ in THF) was added. The reaction was allowed to stir at this temperature for 48 hours. Upon completion, the reaction mixture was filtered through a plug of silica, eluted with $\mathrm{Et}_{2} \mathrm{O}$ and concentrated in vacuo. Purification by silica gel 
chromatography provided (10 to $20 \% \mathrm{Et}_{2} \mathrm{O} /$ pentane gradient) the title compound as clear oil (106 mg, 99\% yield, 10:1 syn/anti). Syn-disatereomer: IR (film) 2959, 2875, 1740, 1707, 1457, 1438, 1393, 1368, 1319, $1171 \mathrm{~cm}^{-1} ;{ }^{1} \mathrm{H}$ NMR (300 MHz, $\left.\mathrm{C}_{6} \mathrm{D}_{6}\right) \delta 0.83(\mathrm{t}, 3 \mathrm{H}$, $\left.J=7.5 \mathrm{~Hz}, \mathrm{CH}_{3}\right), 1.12\left(\mathrm{~m}, 2 \mathrm{H}, \mathrm{CH}_{2}\right), 1.30\left(\mathrm{~m}, 2 \mathrm{H}, \mathrm{CH}_{2}\right), 1.38$ (s, 9H, OC(CH$\left.)_{3}\right), 2.06-$ $2.17(\mathrm{~m}, 1 \mathrm{H}, \mathbf{H C H}), 2.18\left(\mathrm{dd}, 1 \mathrm{H}, J_{l}=6.8\right.$ and $\left.J_{2}=16 \mathrm{~Hz}, \mathrm{HCHCO}_{2} \mathrm{CH}_{3}\right), 2.51(\mathrm{dd}, 1 \mathrm{H}$, $J_{I}=5.9$ and $\left.J_{2}=15.8 \mathrm{~Hz}, \mathbf{H C H C O} \mathrm{CH}_{3}\right), 3.21\left(\mathrm{~s}, 3 \mathrm{H}, \mathrm{CO}_{2} \mathrm{CH}_{3}\right), 4.15(\mathrm{~m}, 2 \mathrm{H}, \mathrm{NCH}$ and OCH) ppm; ${ }^{13} \mathrm{C}$ NMR $\left(75 \mathrm{MHz}, \mathrm{C}_{6} \mathrm{D}_{6}\right) \delta 13.9,20.0,28.2,37.6,38.8,40.6,51.2,60.4$, 77.2, 80.8, 158.8, $169.0 \mathrm{ppm}$; HRMS (EI) exact mass calculated for $\left(\mathrm{C}_{14} \mathrm{H}_{25} \mathrm{NO}_{5}\right)$ requires $m / z[\mathrm{M}+] 287.1733$, found $m / z$ 287.1725. $[\alpha]_{D}^{25}=+39.3^{\circ}\left(\mathrm{c}=0.23, \mathrm{CHCl}_{3}\right)$.

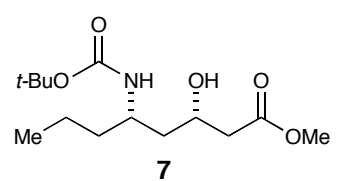

(2S,4S)-tert-Butyl-1-(methoxycarbonyl)-2-hydroxyheptan-4-ylcarbamate (7): An oven-dried $10 \mathrm{~mL}$ round-bottom flask charged with $6(58 \mathrm{mg}, 0.20 \mathrm{mmol})$ in degassed $\mathrm{MeOH}(1 \mathrm{~mL})$ was added $\mathrm{SmI}_{2}(6 \mathrm{~mL}, 0.1 \mathrm{M}$ in THF) under an argon atmosphere. The deep-blue solution was allowed to stir at ambient temperature until the reaction turned pale-yellow (1 hour) or deemed complete by TLC. The solvent was removed in vacuo then the residue re-suspended in dichloromethane $(20 \mathrm{~mL})$. The organic layer was washed successively with aqueous $1 \mathrm{M} \mathrm{NaHSO}_{4}, \mathrm{H}_{2} \mathrm{O}$, brine, dried over $\mathrm{MgSO}_{4}$, and concentrated in vacuo. Purification by silica gel chromatography provided (10 to 30\% EtOAc/hexanes gradient) the title compound as clear oil (58 mg, 99\% yield). IR (film) 3363, 2959, 2875, 1689, 1525, 1439, 1252, $1172 \mathrm{~cm}^{-1} ;{ }^{1} \mathrm{H}$ NMR (300 MHz, $\left.\mathrm{C}_{6} \mathrm{D}_{6}\right) \delta 0.75$ (t, 3H, $\left.J=6.3 \mathrm{~Hz}, \mathrm{CH}_{3}\right), 0.99-1.13\left(\mathrm{~m}, 4 \mathrm{H}, \mathrm{CH}_{2}\right), 1.32\left(\mathrm{~m}, 2 \mathrm{H}, \mathrm{CH}_{2}\right), 1.38(\mathrm{~s}, 9 \mathrm{H}$, $\left.\mathrm{OC}\left(\mathrm{CH}_{3}\right)_{3}\right), 2.25-2.35\left(\mathrm{~m}, 1 \mathrm{H}, \mathrm{HCHCO}_{2} \mathrm{CH}_{3}\right), 2.40-2.45\left(\mathrm{~m}, 1 \mathrm{H}, \mathbf{H C H C O} \mathrm{CH}_{3}\right), 3.23$ (s, $3 \mathrm{H}, \mathrm{CO}_{2} \mathrm{CH}_{3}$ ), 3.40 (br s, $1 \mathrm{H}, \mathrm{OH}$ ), 3.69 (br s, $1 \mathrm{H}, \mathrm{NH}$ ), 4.00-4.14 (br s, $2 \mathrm{H}, \mathrm{NCH}$ and OCH) ppm; ${ }^{13} \mathrm{C}$ NMR $\left(75 \mathrm{MHz}, \mathrm{CDCl}_{3}\right) \delta 13.7,18.9,28.1,37.8,41.1,42.5,47.9,50.7$, 66.1, 78.3, 155.7, $172.6 \mathrm{ppm}$; HRMS (EI) exact mass calculated for $\left(\mathrm{C}_{14} \mathrm{H}_{27} \mathrm{NO}_{5}\right)$ requires $m / z[\mathrm{M}+\mathrm{H}] 290.1967$, found $m / z$ 290.1977. $[\alpha]_{D}^{25}=+3.81^{\circ}\left(\mathrm{c}=1.38, \mathrm{CHCl}_{3}\right)$. 


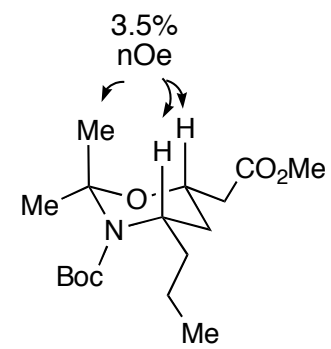

(4S,6S)-tert-Butyl 6-((methoxycarbonyl)methyl)-2,2-dimethyl-4-propyl-1,3oxazinane-3-carboxylate: A half-dram vial charged with $7(10 \mathrm{mg}, 0.034 \mathrm{mmol})$ in DMF $(100 \mu \mathrm{L})$ was added a crystal of $p$ TSA followed by dropwise addition of methoxypropene $(14.3 \mu \mathrm{L}, 0.15 \mathrm{mmol})$. The reaction was stirred at ambient temperature for 1 hour. Upon completion, the reaction was diluted with $\mathrm{Et}_{2} \mathrm{O}(10 \mathrm{~mL})$ and sequentially washed with $\mathrm{H}_{2} \mathrm{O}\left(3 \times \mathrm{X}^{2} \mathrm{~mL}\right)$, brine, then dried over $\mathrm{MgSO}_{4}$ and concentrated in vacuo. Purification by silica gel chromatography (5\% EtOAc/hexanes) provided the title compound as clear oil (9.3 mg, 85\% yield). IR (film) 2959, 1734, 1700, 1689, 1560, $1367 \mathrm{~cm}^{-1}$; ${ }^{1} \mathrm{H}$ NMR (300 MHz, $\left.\mathrm{C}_{6} \mathrm{D}_{6}\right) \delta 0.85\left(\mathrm{t}, 3 \mathrm{H}, J=7.2 \mathrm{~Hz}, \mathrm{CH}_{3}\right), 1.16-$ 1.21 (m, 1H, HCH), 1.27-1.45 (m, 3H, HCHCH $)_{2}, 1.38$ (s, 9H, OC(CH$\left.)_{3}\right), 1.71(\mathrm{~s}, 3 \mathrm{H}$, $\left.\mathrm{CH}_{3}\right), 1.79\left(\mathrm{~s}, 3 \mathrm{H}, \mathrm{CH}_{3}\right), 1.80-1.90\left(\mathrm{~m}, 2 \mathrm{H}, \mathrm{CH}_{2}\right), 2.10\left(\mathrm{dd}, 1 \mathrm{H}, J_{1}=5.4\right.$ and $J_{2}=15.8 \mathrm{~Hz}$, $\left.\mathrm{HCHCO}_{2} \mathrm{CH}_{3}\right), 2.47\left(\mathrm{dd}, 1 \mathrm{H}, J_{1}=8.4\right.$ and $\left.J_{2}=15.8 \mathrm{~Hz}, \mathbf{H C H C O}_{2} \mathrm{CH}_{3}\right), 3.27(\mathrm{~s}, 3 \mathrm{H}$, $\left.\mathrm{CO}_{2} \mathrm{CH}_{3}\right), 4.27$ (m, 2H, NCH and OCH) ppm; ${ }^{13} \mathrm{C} \mathrm{NMR}\left(75 \mathrm{MHz}, \mathrm{C}_{6} \mathrm{D}_{6}\right) \delta 13.9,20.0$, 24.9, 28.1, 28.6, 32.3, 40.2, 42.2, 49.6, 50.7, 63.0, 78.7, 88.1, 153.5, 170.3 ppm; HRMS (EI) exact mass calculated for $\left(\mathrm{C}_{17} \mathrm{H}_{31} \mathrm{NO}_{5}\right)$ requires $\mathrm{m} / \mathrm{z}[\mathrm{M}+\mathrm{H}] 330.2280$, found $\mathrm{m} / \mathrm{z}$ 330.2289. $[\alpha]_{D}^{25}=-29.2^{\circ}\left(\mathrm{c}=0.40, \mathrm{CHCl}_{3}\right)$. The relative syn-configuration of the $\mathbf{H}-\mathrm{CN}$ and $\mathbf{H}-\mathrm{CO}$ protons was determined by irradiation of the $4.27 \mathrm{ppm}$ peak which resulted in a $\mathrm{nOe}(3.5 \%)$ on the $1.71 \mathrm{ppm}$ singlet.
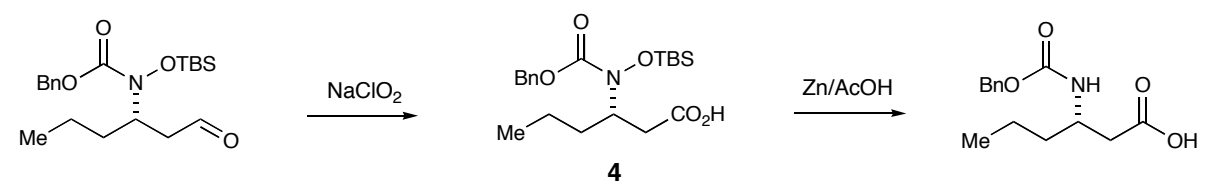

Determination of Absolute Configuration by Chemical Correlation: $N$-tertbutyldimethylsilyloxy[benzyl-(S)-1-formylpentan-2-ylcarbamate] (Table 2, entry 2) was derivatized to the $\beta$-amino acid by Pinnick oxidation according to procedure described for compound 4. Removal of the silyl-protecting group and reduction of $\mathrm{N}-\mathrm{O}$ bond was 
accomplished in a single step with acetic acid and Zn dust to afford benzyl (S)-3carbamate-hexanoic acid. All spectroscopic data matched literature reported values. ${ }^{2}$ The observed optical rotation $\left([\alpha]_{D}^{25}=-11.45^{\circ}(\mathrm{c}=0.50, \mathrm{MeOH})\right)$ was consistent with the $(S)$ configuration for benzyl (S)-3-carbamate-hexanoic acid, based on the previously reported $(R)$-configuration ${ }^{3}$ having the opposite sign of rotation $\left([\alpha]_{D}^{25}=+12.9^{\circ}(\mathrm{c}=0.55\right.$, $\mathrm{MeOH})$ ).

1. Pangborn, A. B.; Giardello, M. A.; Grubbs, R. H.; Rosen, R. K.; Timmers, F. J. Organometallics 1996, 15, 1518-1520.

2. Braschi, I.; Cardillo, G.; Tomasini, C.; Venezia, R. J. Org. Chem. 1994, 59, 72927298.

3. $\quad$ Seki, M.; Matsumoto, K. Tetrahedron Lett. 1996, 37, 3165-3168. 


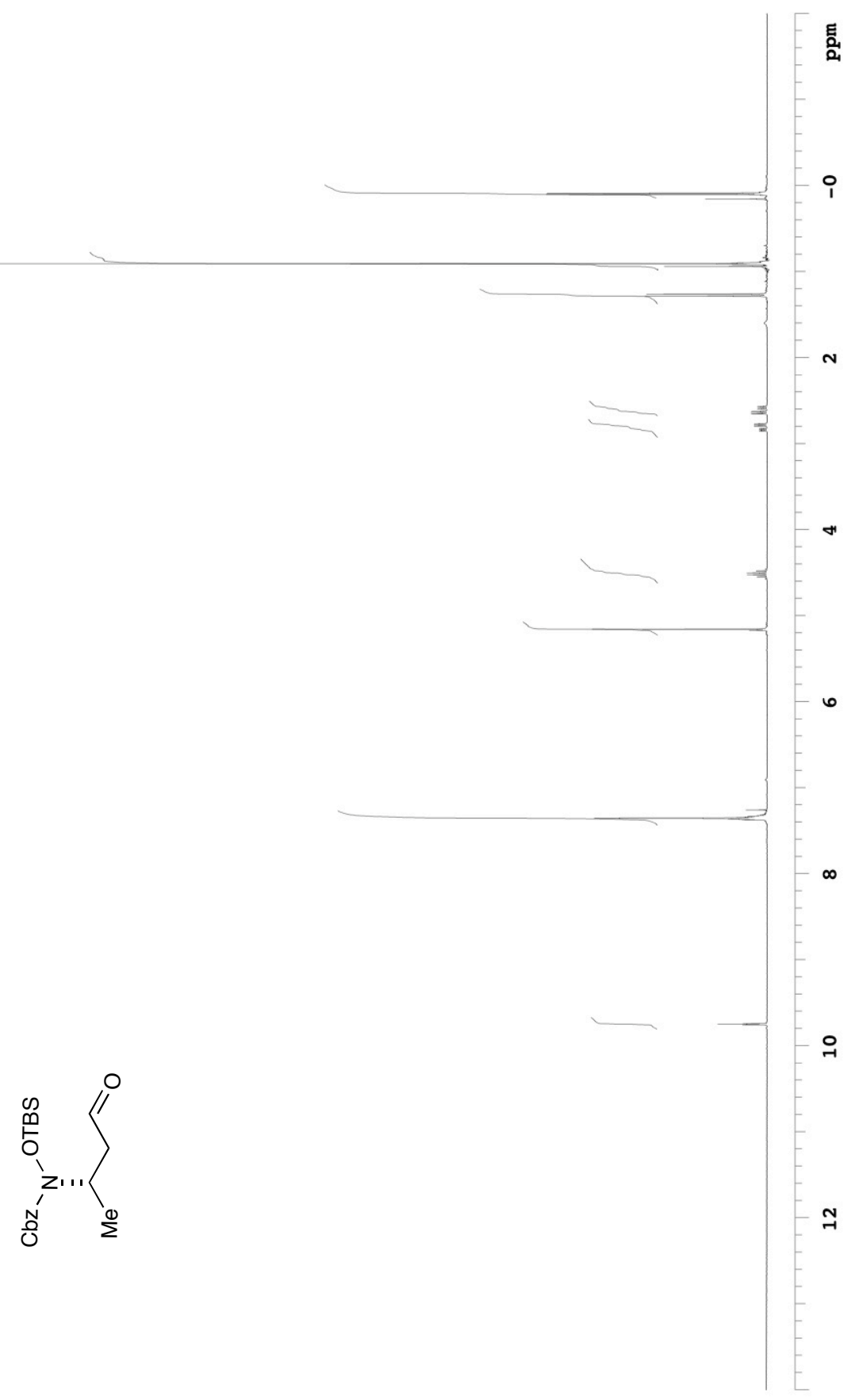




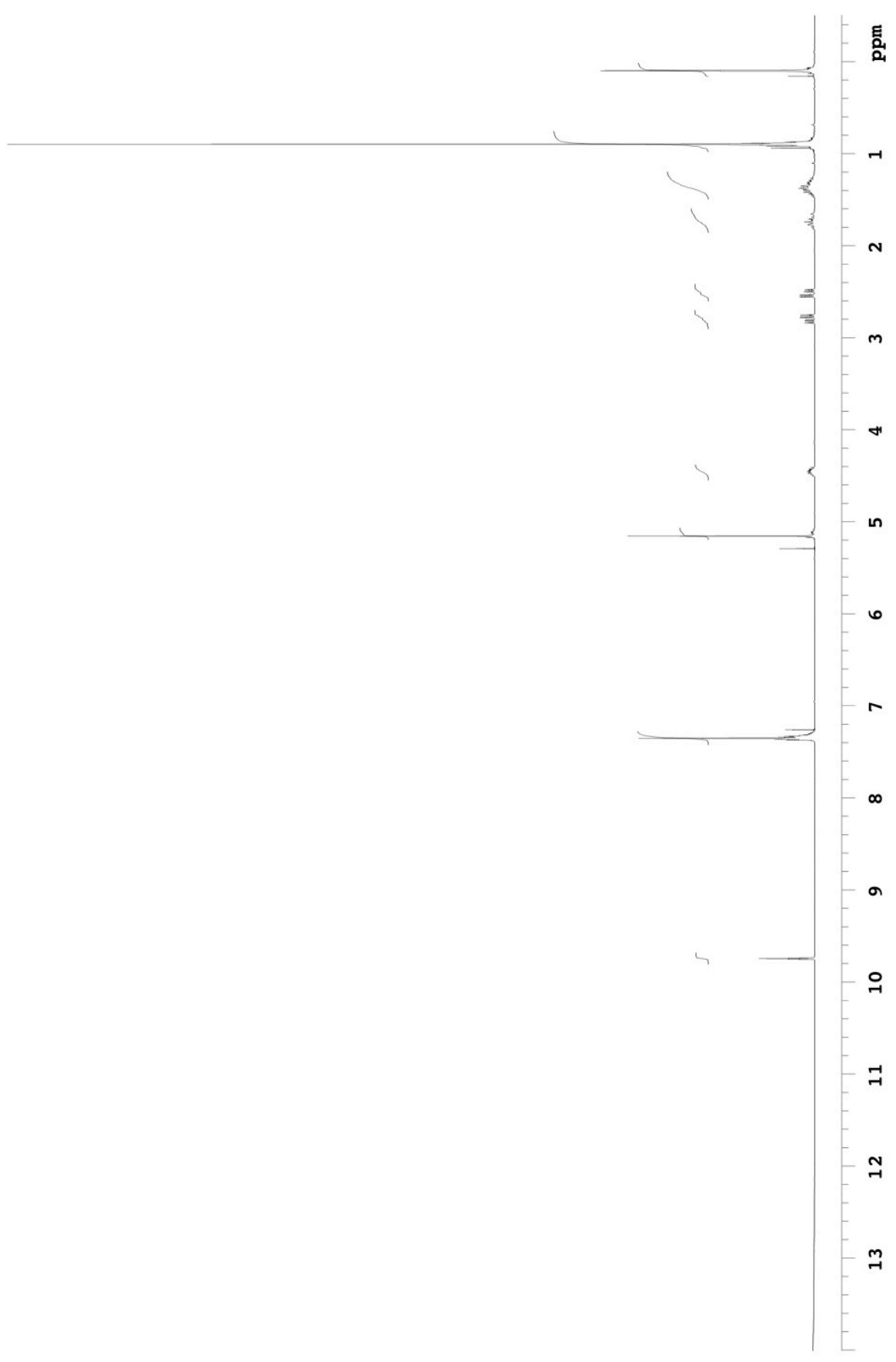




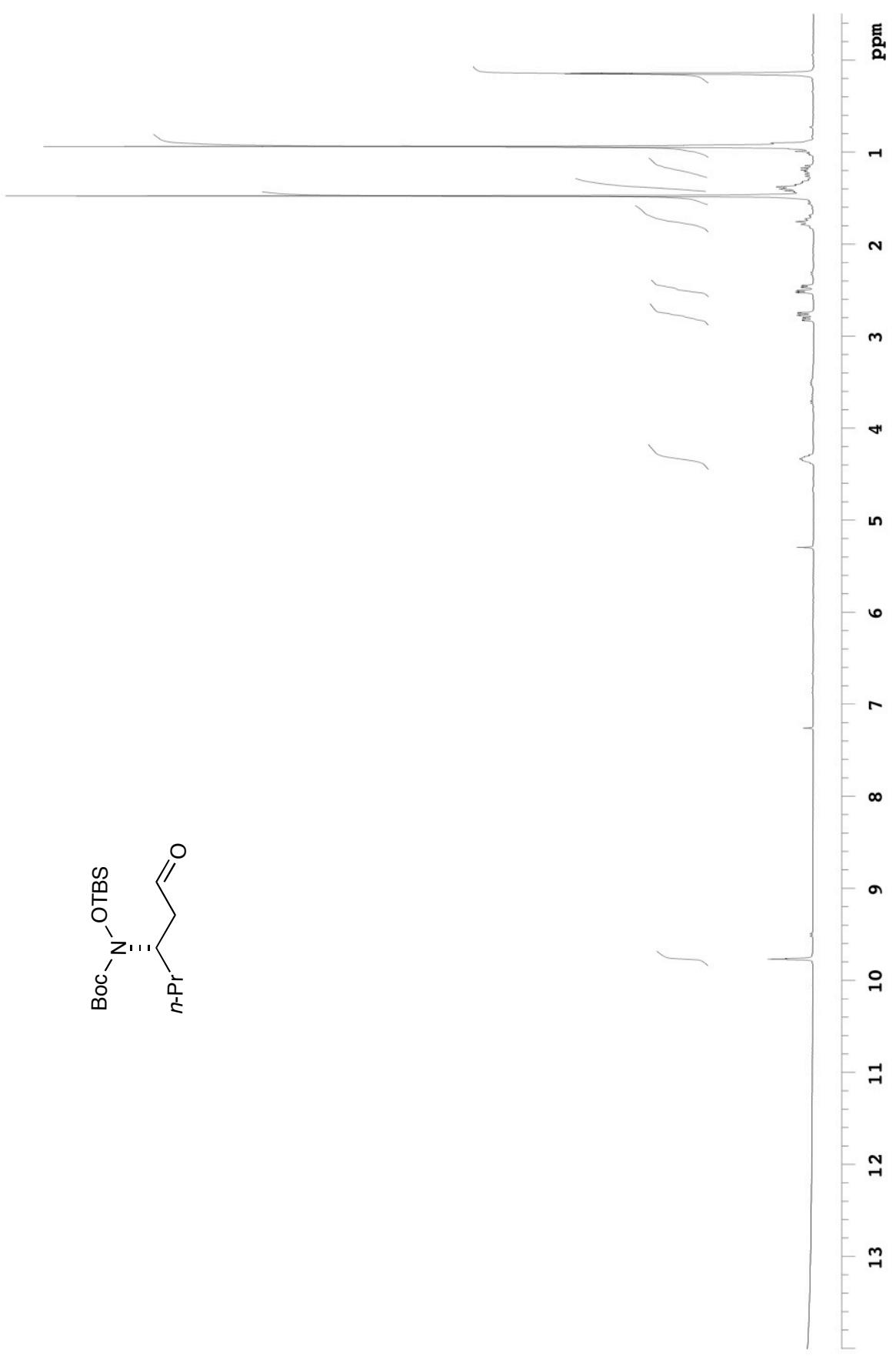




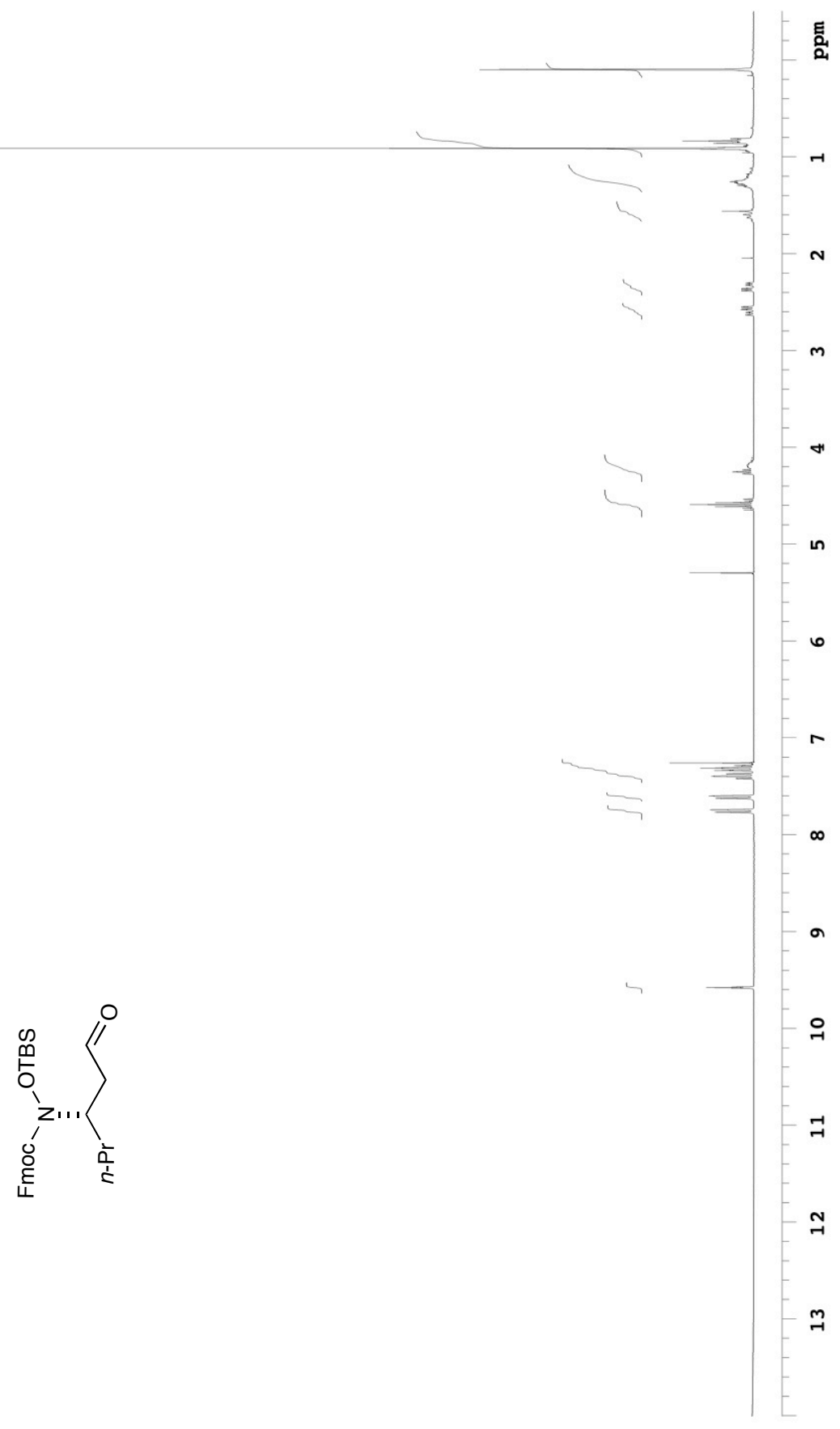




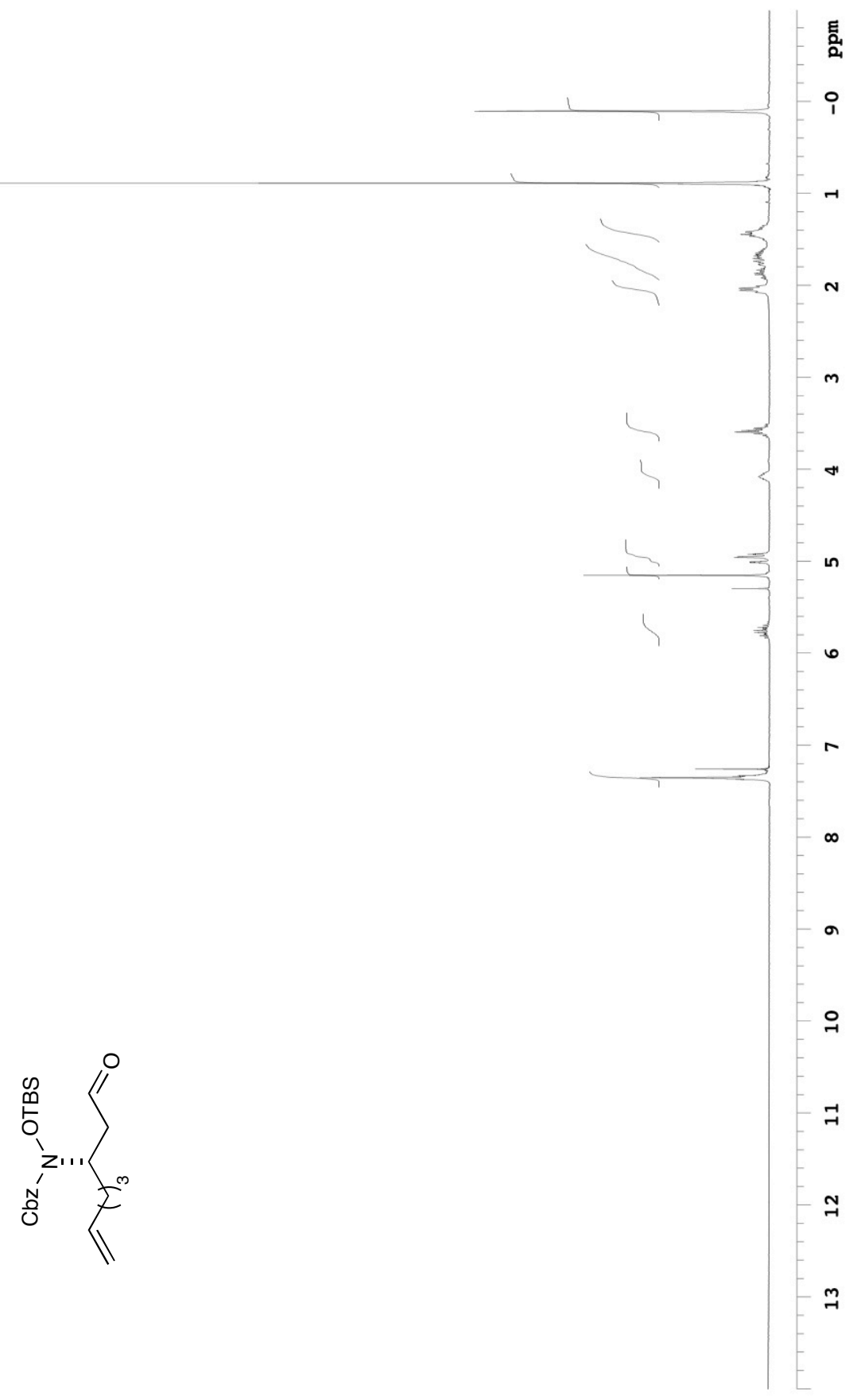




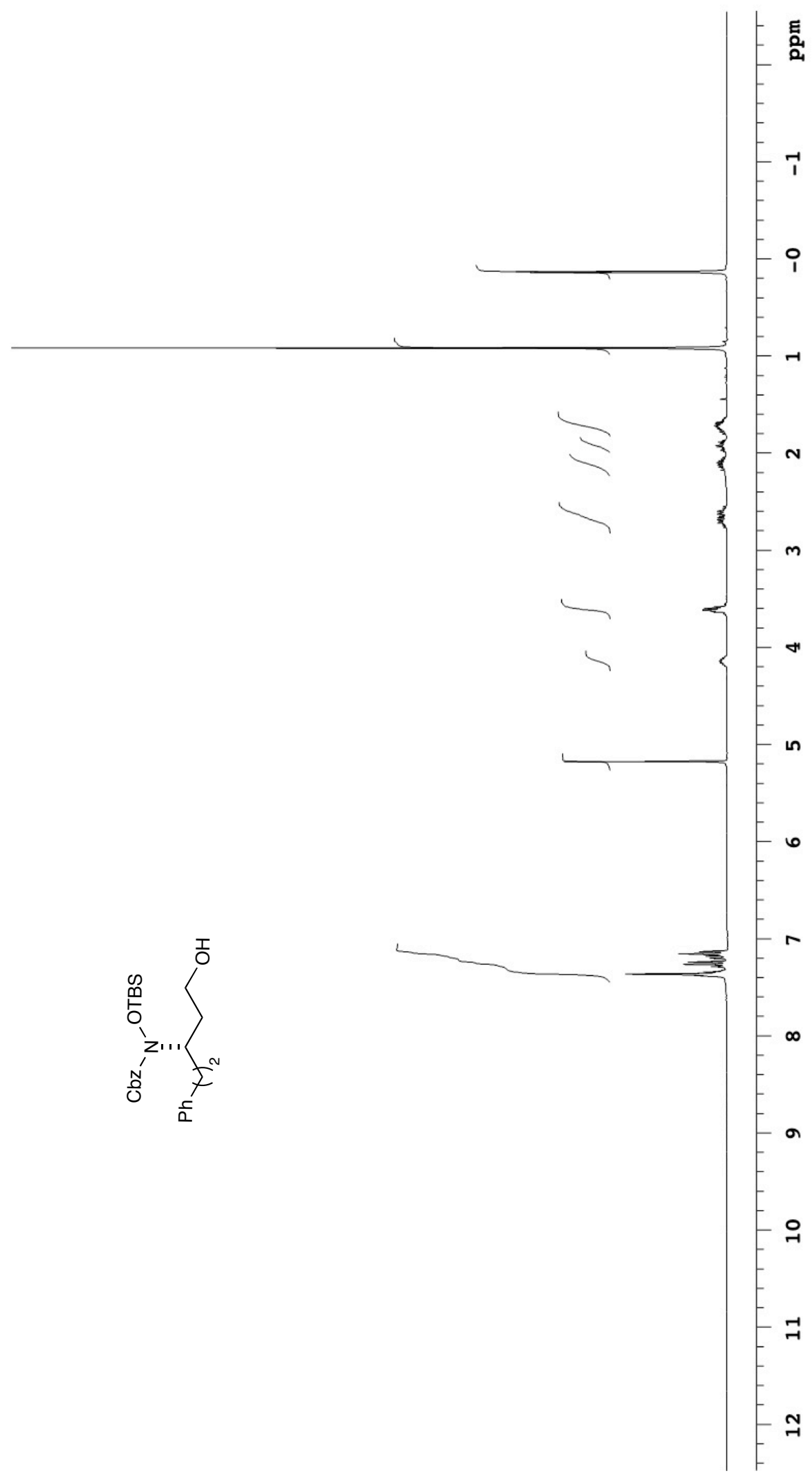




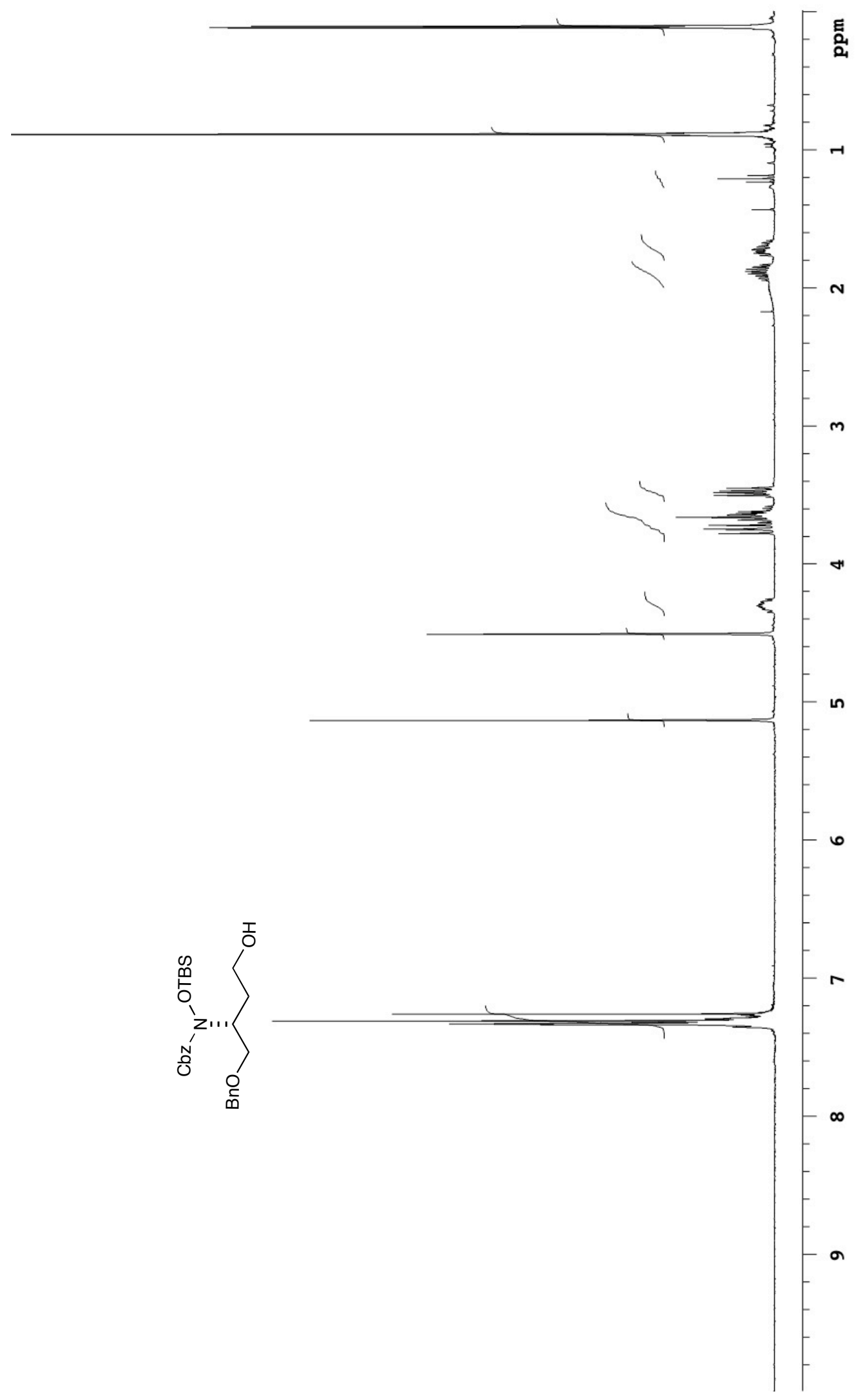




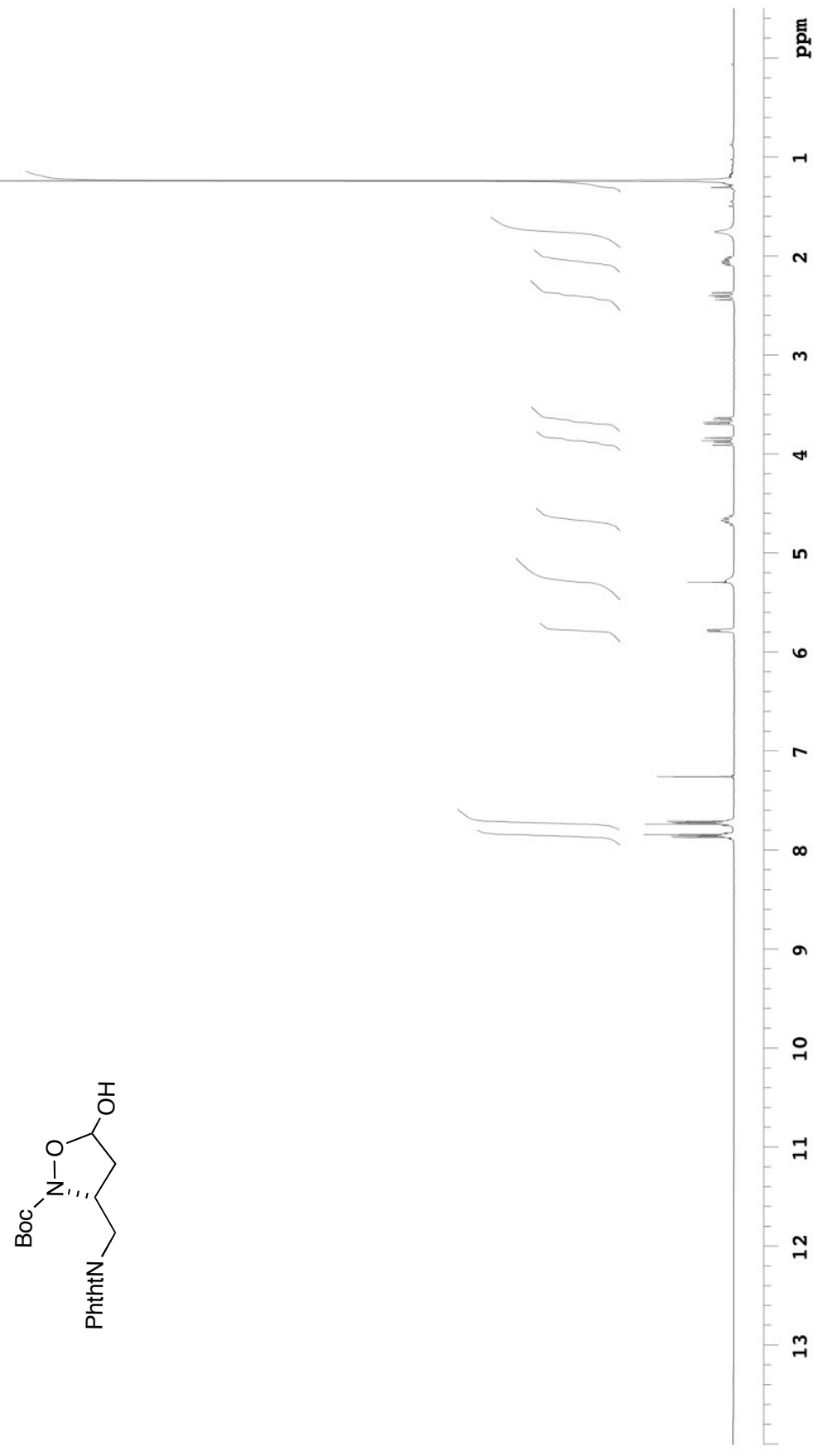




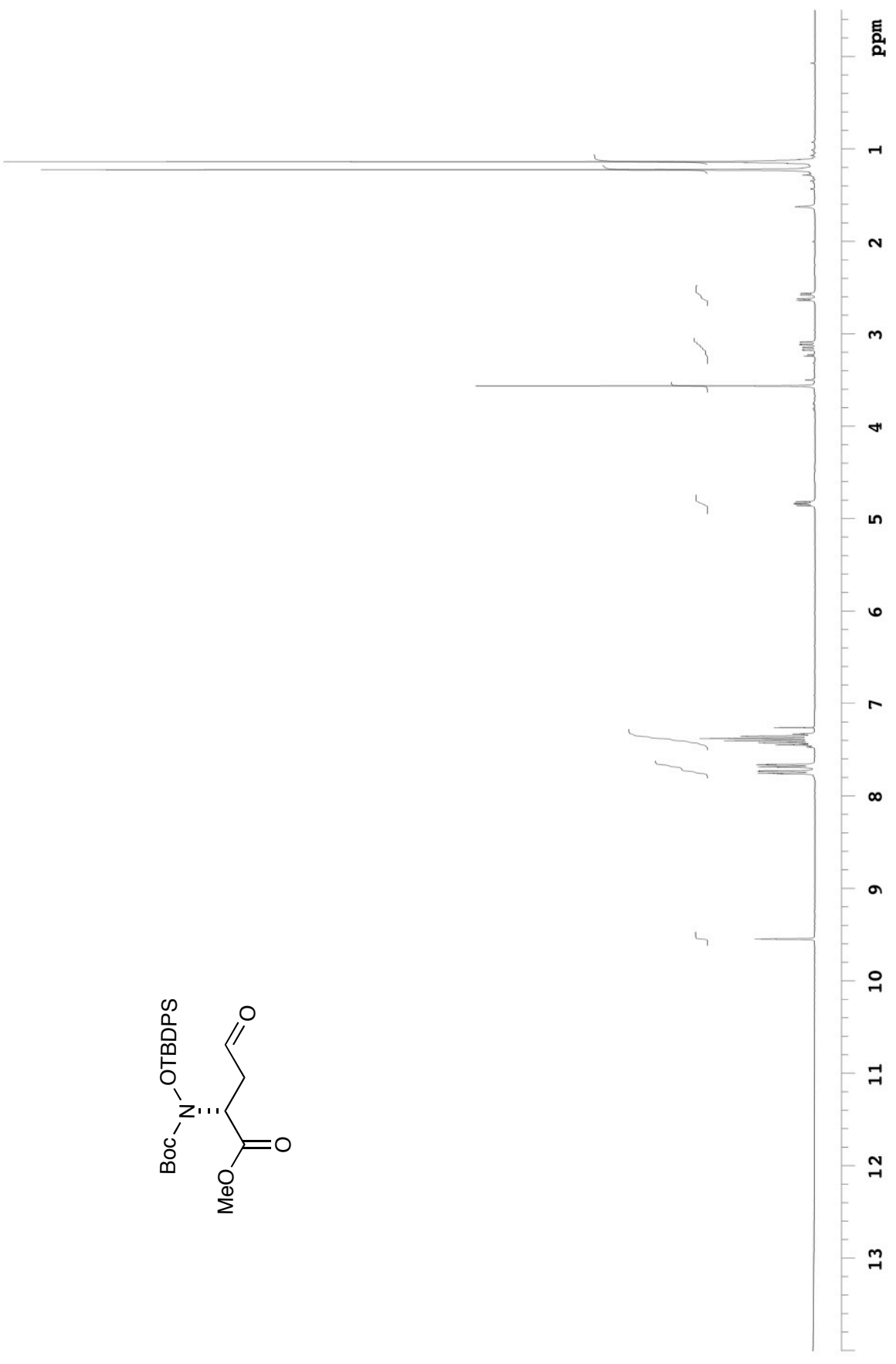




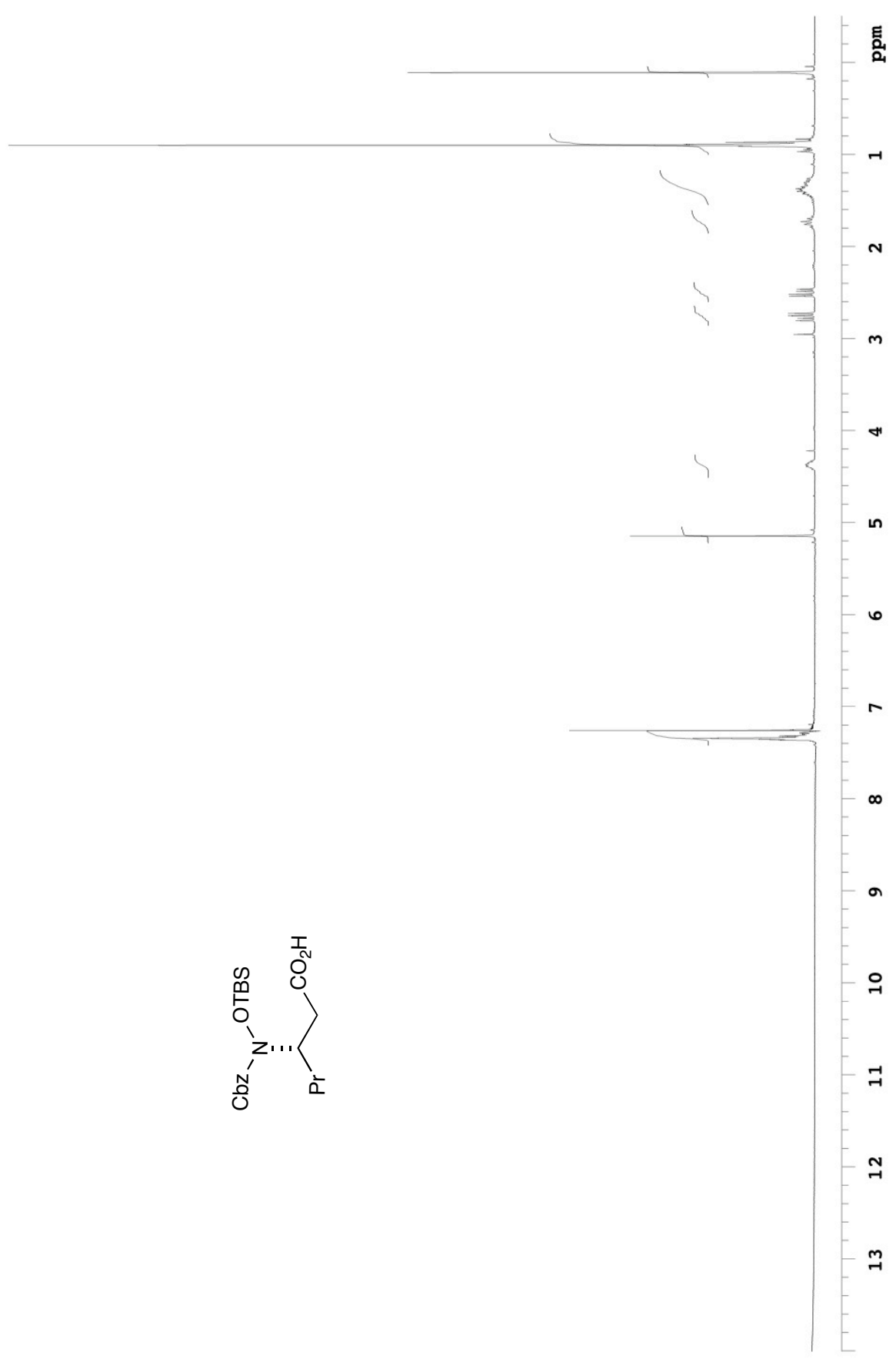




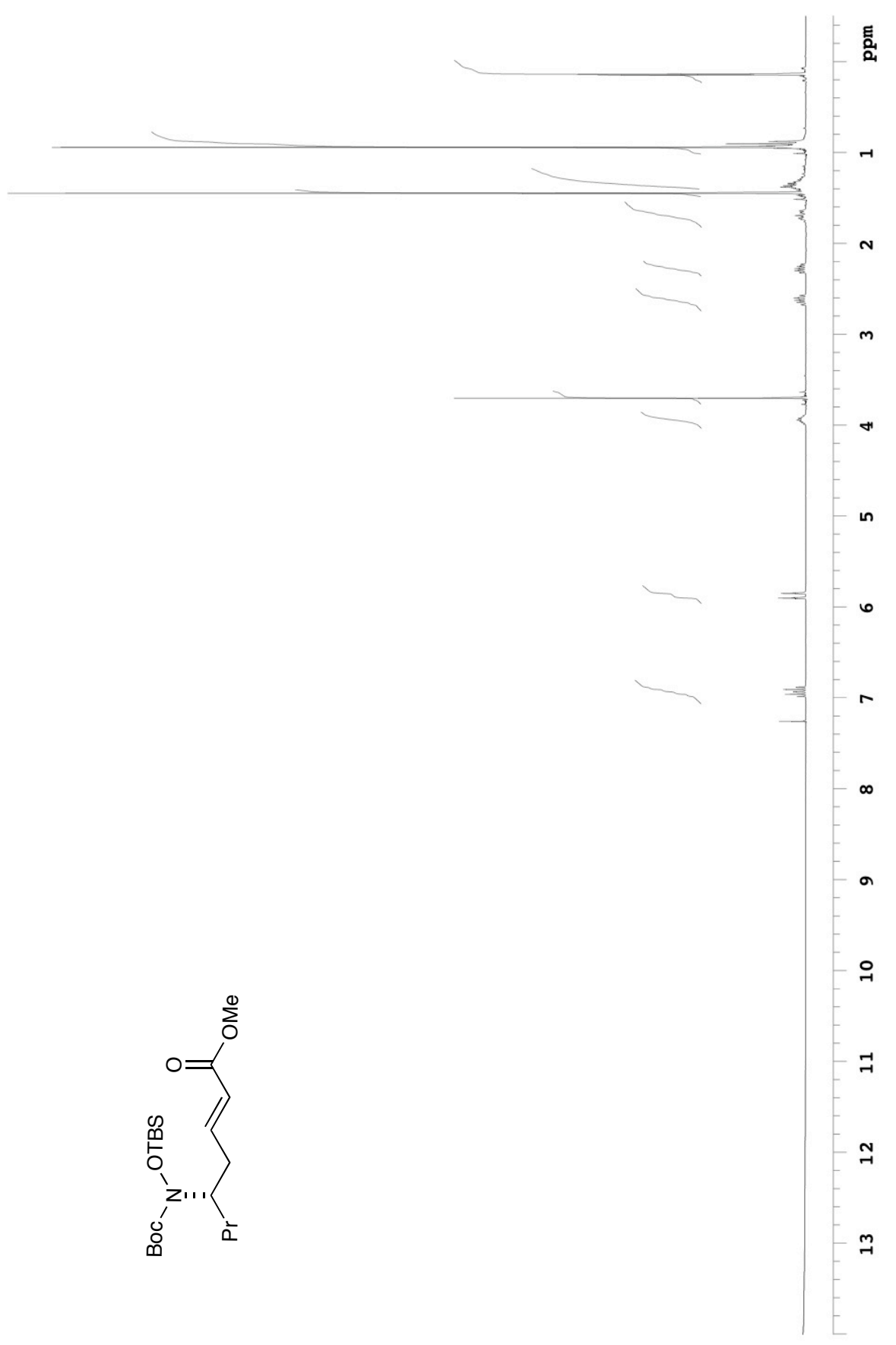




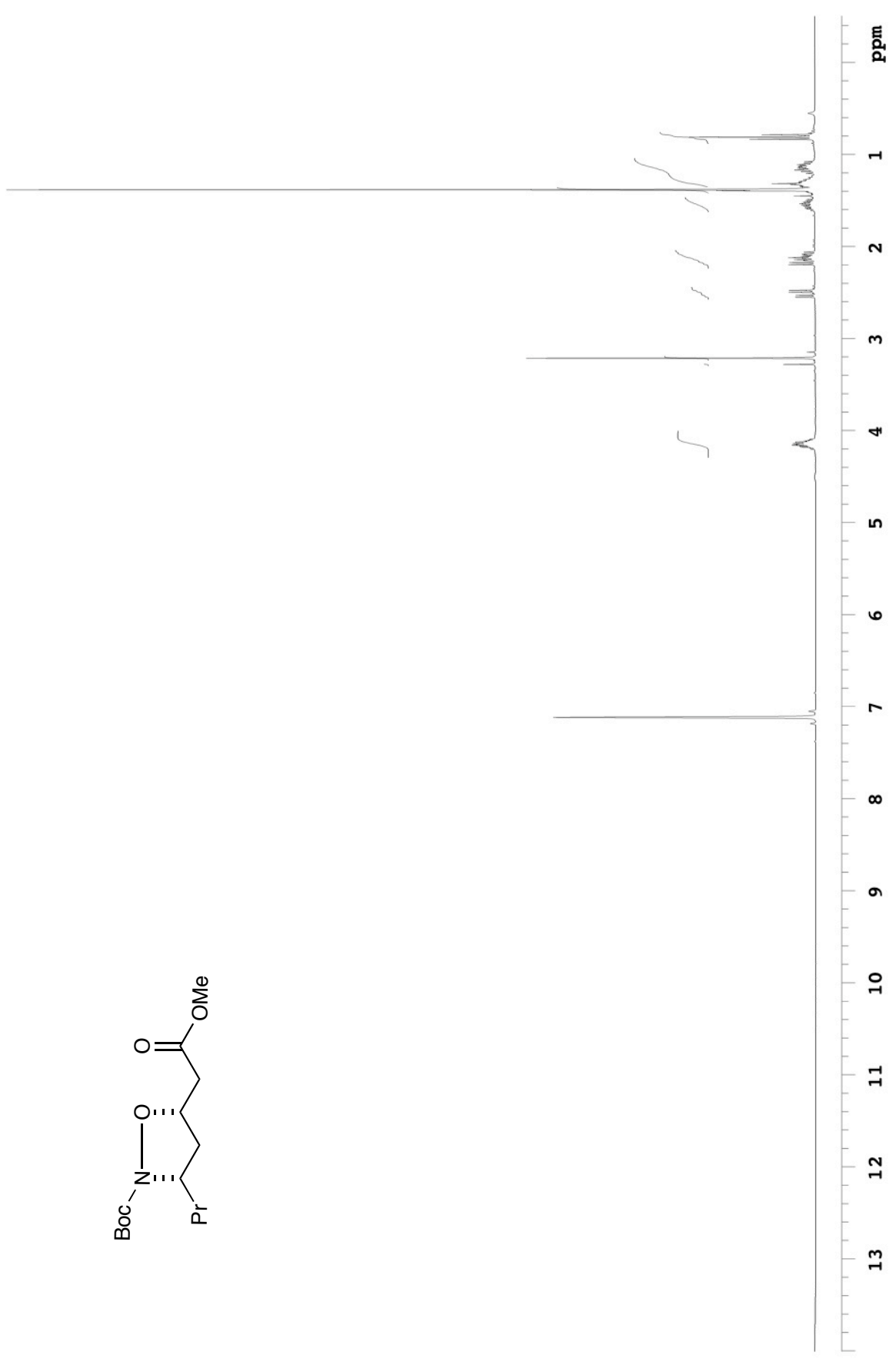




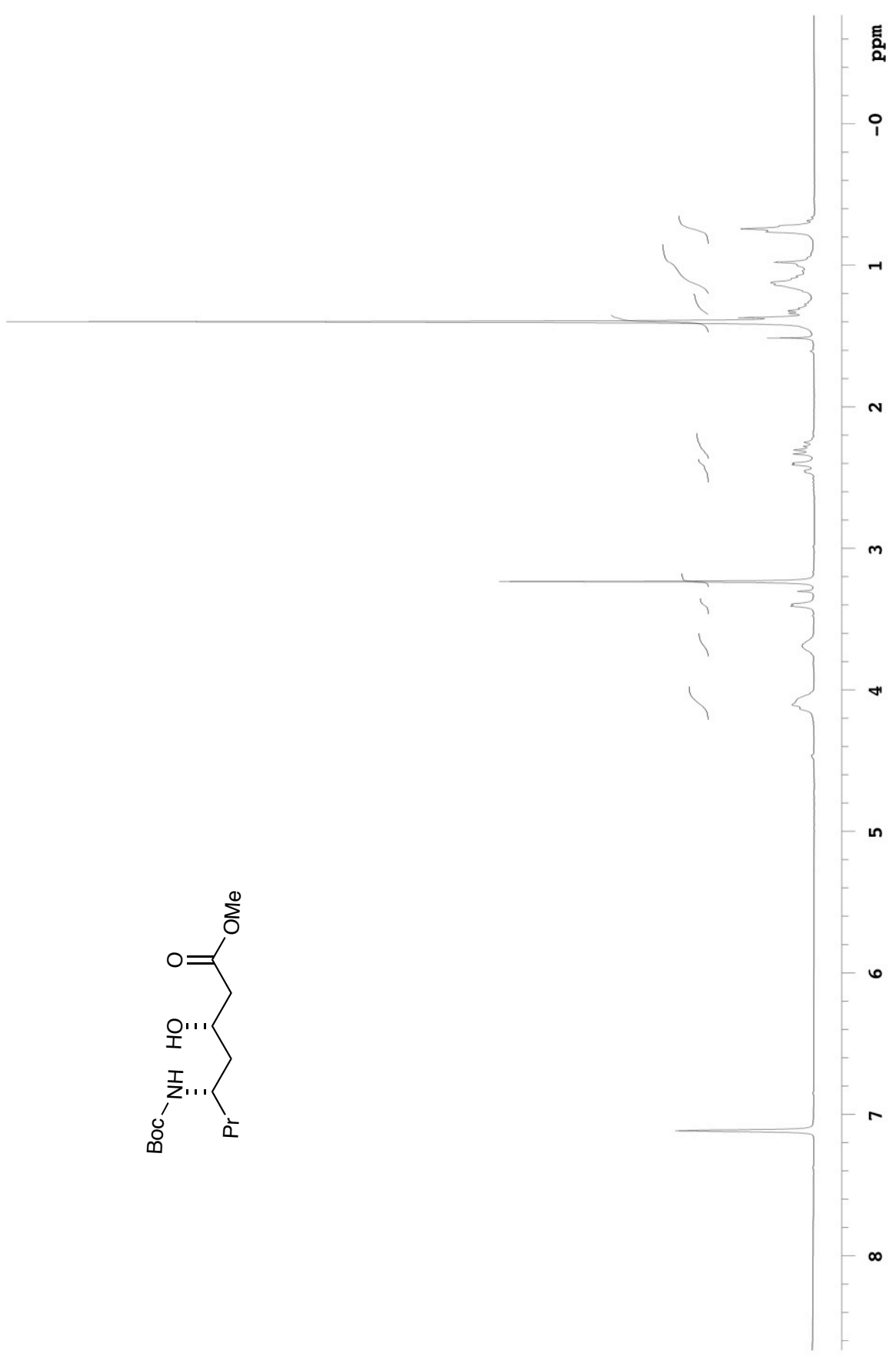




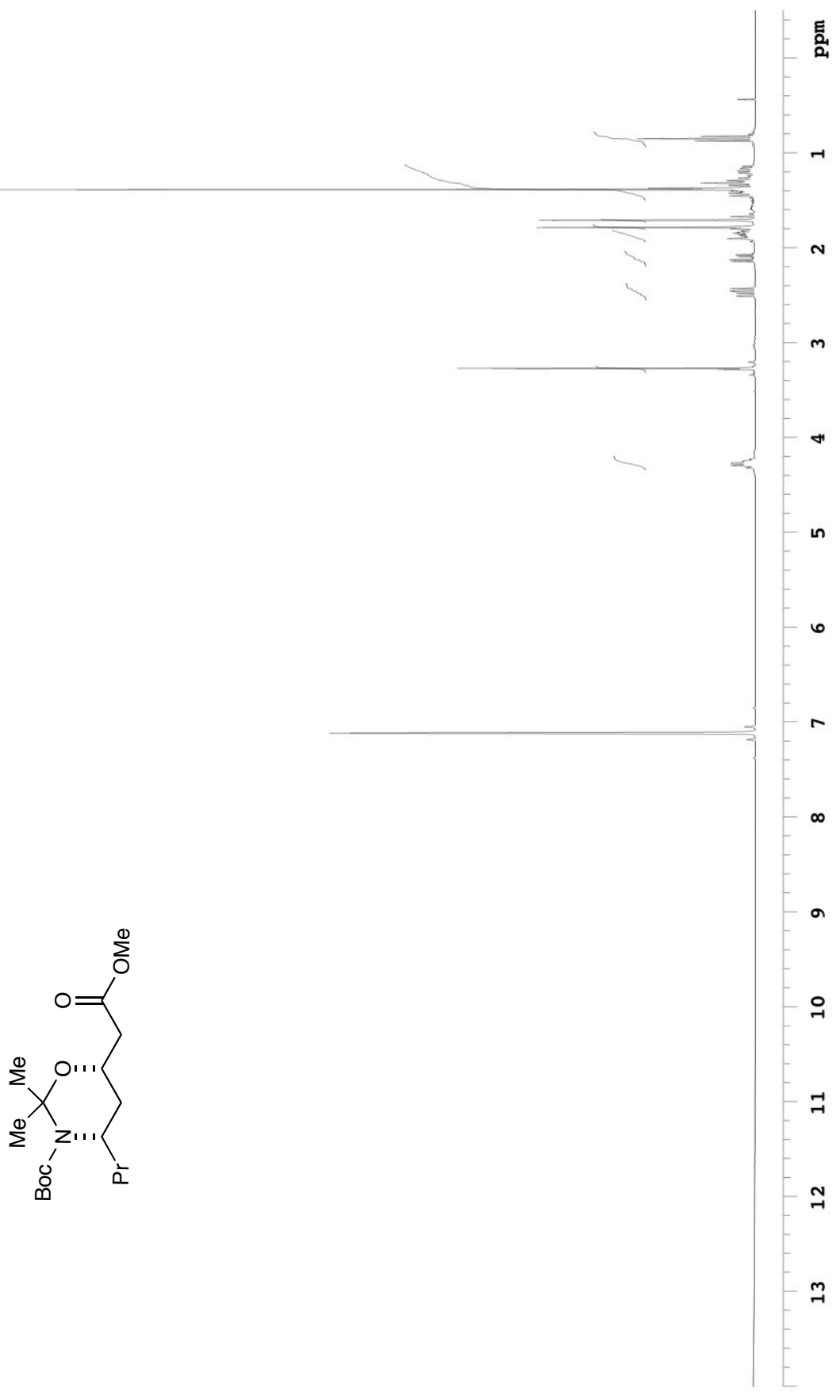


